\title{
PARAREAL OPERATOR SPLITTING TECHNIQUES FOR MULTI-SCALE REACTION WAVES: NUMERICAL ANALYSIS AND STRATEGIES
}

\author{
Max Duarte $^{1}$, Marc Massot $^{1}$ and Stéphane Descombes ${ }^{2}$
}

\begin{abstract}
In this paper, we investigate the coupling between operator splitting techniques and a time parallelization scheme, the parareal algorithm, as a numerical strategy for the simulation of reactiondiffusion equations modelling multi-scale reaction waves. This type of problems induces peculiar difficulties and potentially large stiffness which stem from the broad spectrum of temporal scales in the nonlinear chemical source term as well as from the presence of large spatial gradients in the reactive fronts, spatially very localized. In a series of previous studies, the numerical analysis of the operator splitting as well as the parareal algorithm has been conducted and such approaches have shown a great potential in the framework of reaction-diffusion and convection-diffusion-reaction systems. However, complementary studies are needed for a more complete characterization of such techniques for these stiff configurations. Therefore, we conduct in this work a precise numerical analysis that considers the combination of time operator splitting and the parareal algorithm in the context of stiff reaction fronts. The impact of the stiffness featured by these fronts on the convergence of the method is thus quantified, and allows to conclude on an optimal strategy for the resolution of such problems. We finally perform some numerical simulations in the field of nonlinear chemical dynamics that validate the theoretical estimates and examine the performance of such strategies in the context of academical one-dimensional test cases as well as multi-dimensional configurations simulated on parallel architecture.
\end{abstract}

Mathematics Subject Classification. 65Y05, 65M12, 65L04, 35A35, 35K57, 35C07.

Received May 6, 2010.

Published online February 23, 2011.

\section{INTRODUCTION}

Numerical simulations of multi-scale phenomena are commonly requested for modelling purposes in many applications such as combustion [10,31,40,49,50], chemical vapor deposition [32], or air pollution modelling [39, 48,61]. The important development of the numerical strategies in these and in other fields such as nonlinear chemical dynamics for excitable media $[6,20,21]$ or biomedical engineering $[12,22,34]$ is mainly due to the constant increase of the computer power (see for instance [23] for a recent review of methods applied to turbulent combustion). In general, all these models raise several difficulties created by the large number of unknowns and the wide range of temporal scales due to large and detailed chemical kinetic mechanisms, as well as by steep

\footnotetext{
Keywords and phrases. Parareal algorithm, operator splitting, convergence analysis, reaction-diffusion, multi-scale waves.

${ }^{1}$ Laboratoire EM2C - UPR CNRS 288, École Centrale Paris, Grande Voie des Vignes, 92295 Chatenay-Malabry Cedex, France. max.duarte@em2c.ecp.fr; marc.massot@em2c.ecp.fr

${ }^{2}$ Laboratoire J.A. Dieudonné - UMR CNRS 6621, Université de Nice Sophia Antipolis, Parc Valrose, 06108 Nice Cedex 02, France. sdescomb@unice.fr
} 
spatial gradients or large higher order derivatives associated with very localized fronts of high chemical activity. Therefore, there are several numerical strategies in order to treat the induced stiffness for time dependent problems. In this particular study, we focus on reaction-diffusion systems which is the subsystem that normally involves the strongest difficulties in terms of stiffness in multi-scale phenomena, even if convection plays also a crucial role.

The most natural idea to overcome these difficulties is to use dedicated numerical methods and to solve the complete models where diffusion, reaction and eventually convection are coupled together. In this context, we aim at solving strongly coupled nonlinear systems with either a fully implicit method, using eventually modified Newton methods for ill-conditioned problems [18,19,53], or yet semi-implicit or linearized implicit methods instead (see [8] and references therein). However, the strong stability restrictions for the latter when dealing with very fast temporal scales [8,9] as well as the computational cost and the huge memory requirements of these methods, even if adaptive grids are used, make these strategies difficult to handle. Nevertheless, these kind of coupled resolutions are useful when we need reference solutions for validation and study purposes but necessarily restricted to low dimensional configurations with not too many unknowns.

An alternative numerical strategy first introduced in [2] is then to combine implicit and explicit schemes to discretize nonlinear evolution problems in time. Further studies into [54,64] settled the appropriate numerical background for these methods called IMEX, which in particular might be conceived to solve stiff nonlinear problems as presented in $[52,66]$. These methods are usually very efficient (see for instance [50] for a combustion application). Nevertheless, on the one hand, the feasibility of utilizing dedicated implicit solvers over a discretized domain become soon critical when treating large computational domains. And on the other hand, the time steps globally imposed over partial regions or the entire domain are strongly limited by either the stability restrictions of the explicit solver or by the fastest scales treated by the implicit scheme.

Nevertheless, in many multi-scale problems as for example the propagation of reaction waves, the fastest time scales do not play a leading role in the global physical phenomenon and thus, we might consider the possibility of using reduced models where these chemical scales have been previously relaxed [35]. These simplified models provide reasonable predictions when the fastest characteristic chemical times are small in comparison with the flow time, and the associated computational costs are significantly reduced in comparison with comprehensive chemical models. In particular, the derivation of the reduced model is usually accessible when the system is well-partitioned and the fast scales have been isolated [55,57]. In this case, a rigorous singular perturbation analysis can be conducted even in the context of nonlinear source terms for numerical analysis purposes [13,47]. Nevertheless, the identification of these fast scales in terms of reaction rates or species, which can change with time, relies on sensitivity analysis which is most of the time difficult to conduct and justify in realistic configurations. Hence, it reveals the need for other strategies which do not rely on the knowledge of the fast scales.

It is then natural to envision a compromise, since the fully coupled problem is most of the time out of reach and the reduced model does not always imply straightforward implementations. In this context, splitting methods [46] also called fractional step methods $[62,63,68]$ have been well known for a long time and there exists a large literature showing the efficiency of such methods for evolution problems. Yet from a theoretical point of view, they represent a suitable framework to design even higher order methods for the integration in time of such problems $[11,51]$. In practice, it is firstly necessary to decouple numerically the reaction part from the rest of the physical phenomena like convection, diffusion or both, for which there also exist dedicated numerical methods. Hence, operator splitting techniques allow a completely independent optimization of the resolution of each subsystem which normally yields lower requirements of computational resources.

In the context of multi-scales waves, the dedicated methods chosen for each subsystem are then responsible for dealing with the fast scales associated to each one of them, in a separate manner; then, the composition of the global solution based on the splitting scheme should guarantee the good description of the global physical coupling; therefore, in order to verify this fundamental constraint, a rigorous numerical analysis is required. In fact, several works $[8,35,56,65]$ proved that the classical numerical analysis of splitting schemes fails in presence of scales much faster than the splitting time step and motivated more rigorous studies for these stiff 
configurations [13]. In this way, complementary works described also the numerical behavior of these methods when spatial multi-scale phenomena arise mainly as a consequence of large spatial gradients [16], so that the influence of both spatial and time related stiffness has been and continues to be analyzed in detail for not arbitrarily small splitting time steps [17].

Thus, with the choice of the resolution technique properly justified, we investigate the coupling of operator splitting with a time parallelization scheme, pursuing even better performances in time consumption for multiscale simulations on parallel architectures. In this context, many algorithms already proposed the solution of evolution problems in a time-parallel fashion (see [28] and the references therein for a historical review). However, the parareal algorithm, first presented in [42], has received a lot of attention over the past few years in different applications in different domains [3,5,25,26,29], as a promising efficient numerical method to solve evolution problems in parallel. The general principle of the parareal algorithm combines a coarse and fast solver which is run sequentially, and a more accurate and expensive fine solver that should be run in parallel. In this way, we take advantage of parallel computations through an iterative process which yields convergence from a coarse initial approximation to the detailed dynamics of the system given by the accurate resolution of the fine solver. Several variants of the method have been also proposed (for example in $[25,30]$ ).

Up to these days, many theoretical analysis of the parareal algorithm have been conducted (see for instance $[4,27,28,42,44,45,58])$, which led to various estimates of convergence rates and descriptions of the stability behavior of the method in applications involving general linear and nonlinear systems of ODEs or PDEs. Nevertheless, to our knowledge, none of the studies previously conducted either in a linear or in a nonlinear framework, took explicitly into account stiff phenomena. As a result, there is the need of complementary studies to utterly predict the performance of the algorithm in such configurations in order to propose more efficient time parallelization schemes in multi-scale contexts.

Keeping this in mind, the present work conducts a detailed numerical analysis of parareal operator splitting techniques in the context of multi-scale reaction waves. In this way, new representation of the splitting local errors are deduced and a convergence analysis of the parareal operator splitting algorithm is performed for general linear reaction-diffusion systems. These results are then extended to the case of reaction waves through the associated linearized system of the original reaction-diffusion system modelling the multi-scale phenomenon, where stiffness is introduced by the presence of large spatial gradients. The negative influence of stiffness on the numerical behavior of parareal operator splitting schemes is then mathematically proven.

With this theoretical characterization achieved, we are able to build a new and simple numerical strategy for multi-scale phenomena, with the parareal algorithm and the operator splitting as fine solver, each one of them based on their corresponding theoretical background. The time operator splitting strategy adopted then considers on the one hand, a high order method like Radau5 [35], based on implicit Runge-Kutta schemes for stiff ODEs, that solves the reaction term using adaptive time integration tools and highly optimized linear systems solvers. And on the other hand, another high order method like ROCK4 [1], based on explicit stabilized RungeKutta schemes, that solves the diffusion problem. The potential of this splitting configuration has already been tested and evaluated in previous studies $[21,22]$. The numerical simulations then performed show the influence of a stiff configuration on the parareal performance, validating the theoretical estimates previously conducted. Finally, the discussions inspired by these numerical results gives some insights into alternative numerical strategies for this kind of difficult problems.

The paper is organized as follows: in a first part, we formally present the parareal algorithm as well as the splitting techniques which will be used in the proposed numerical strategy. The complete numerical analysis of the parareal operator splitting is conducted in the second part: a brief review on the theoretical results found in the literature is first presented, then the different local error estimates are carefully conducted to finish with the theoretical convergence analysis in a general linear reaction-diffusion configuration and in the case of multi-scale reaction waves through a linearized model of such configurations. In the last part, we first conduct a series of detailed and careful numerical simulations in a one-dimensional case in order to validate the previous theoretical results. Finally, the potential of the method is illustrated in the framework of a two-dimensional simulation 
which allows a detailed discussion of the capability and performance of the method. All the models belong to the field of nonlinear chemical dynamics.

\section{Parareal algorithm}

\subsection{Temporal parallelization}

We first consider a general method in order to compute the numerical solution of a system of ordinary differential equations (ODEs) of the form:

$$
\mathbf{u}^{\prime}(t)=\mathbf{f}(\mathbf{u}(t)), \quad t \in[0, T], \quad \mathbf{u}(0)=\mathbf{u}^{0},
$$

where for some integer $M, \mathbf{f}: \mathbb{R}^{M} \rightarrow \mathbb{R}^{M}$ and $\mathbf{u}: \mathbb{R} \rightarrow \mathbb{R}^{M}$. In order to achieve a time parallelization algorithm for (1.1), we decompose the time domain $I=[0, T]$ into $N$ subdomains $I_{n}=\left[T_{n}, T_{n+1}[, n=0, \ldots, N-1\right.$, with $0=T_{0}<\ldots<T_{N-1}<T_{N}=T$, and $\Delta T_{n}:=T_{n+1}-T_{n}$, so, that we consider for each time subdomain the evolution problem:

$$
\mathbf{u}_{n}^{\prime}(t)=\mathbf{f}\left(\mathbf{u}_{n}(t)\right), \quad t \in I_{n}, \quad \mathbf{u}_{n}\left(T_{n}\right)=\mathbf{U}_{n},
$$

where the initial values $\mathbf{U}_{n}$ are a necessary input in order to solve each of these evolution problems. The solutions $\mathbf{u}_{n}$ on the subdomains $I_{n}$ in (1.2) should be consistent with $\mathbf{u}$ over $I$ obtained out of (1.1), this means that the initial values $\mathbf{U}_{n}$ are intended to satisfy the system:

$$
\mathbf{U}_{0}=\mathbf{u}^{0}, \quad \mathbf{U}_{n}=\phi^{\Delta T_{n-1}}\left(\mathbf{U}_{n-1}\right) \text { for } n=1, \ldots, N,
$$

where the flow $\phi^{\Delta T_{n}}(\mathbf{U})$ denotes the solution of (1.1) with initial condition $\mathbf{U}$ after time $\Delta T_{n}$.

Thus, we consider $N$ independent evolution problems given by (1.2) for each time subdomain $I_{n}$, so that each of them may be computed by a different processor in a parallel environment. Nevertheless, this can only be achieved if the initial conditions $\mathbf{U}_{n}$ of (1.2) are previously known or at least approximated, in order to have a proper Cauchy problem on each time subdomain. Therefore, any time parallelization algorithm will aim at approximating the initial $\mathbf{U}_{n}$ by the set $\tilde{\mathbf{U}}_{n}$ and then, at solving (1.2), even though condition (1.3) with $\tilde{\mathbf{U}}_{n}$ is not initially verified. In this context, the parareal algorithm gives us a way to perform this kind of parallelization in an efficient way.

This technique extends naturally to the numerical resolution of partial differential equations (PDEs), as it was initially proposed in [42]. In fact, the multi-scale reaction waves that we consider in this study are modeled by means of parabolic PDEs.

\subsection{Principle of the parareal algorithm}

Considering system (1.2), the parareal algorithm is based on two propagation operators: $\mathcal{G}^{\Delta T_{n}}(\mathbf{U})$ and $\mathcal{F}^{\Delta T_{n}}(\mathbf{U})$, that provide respectively a coarse and an accurate approximation of $\phi^{\Delta T_{n}}(\mathbf{U})$. In this way, the algorithm starts with an initial approximation $\tilde{\mathbf{U}}_{n}^{0}$ given for example by the sequential computation:

$$
\tilde{\mathbf{U}}_{0}^{0}=\mathbf{u}^{0}, \quad \tilde{\mathbf{U}}_{n}^{0}=\mathcal{G}^{\Delta T_{n-1}}\left(\tilde{\mathbf{U}}_{n-1}^{0}\right) \text { for } n=1, \ldots, N,
$$

and then performs for $i=1, \ldots, i_{\text {conv }}$ the correction iterations:

$$
\tilde{\mathbf{U}}_{0}^{i}=\mathbf{u}^{0}, \quad \tilde{\mathbf{U}}_{n}^{i}=\mathcal{F}^{\Delta T_{n-1}}\left(\tilde{\mathbf{U}}_{n-1}^{i-1}\right)+\mathcal{G}^{\Delta T_{n-1}}\left(\tilde{\mathbf{U}}_{n-1}^{i}\right)-\mathcal{G}^{\Delta T_{n-1}}\left(\tilde{\mathbf{U}}_{n-1}^{i-1}\right) \text { for } n=1, \ldots, N .
$$

Notice that $i$ iterations imply $i$ time subdomains integrated by $\mathcal{F}$, since $\tilde{\mathbf{U}}_{n}^{i}=\mathcal{F}^{\Delta T_{n-1}}\left(\tilde{\mathbf{U}}_{n-1}^{i-1}\right)$ for $n \leq i$ and $\tilde{\mathbf{U}}_{0}^{i}=\mathbf{u}^{0}$ with $\mathbf{u}^{0}$ taken from the original problem (1.1). Then, for $i=N$, where $N$ is the number of subdomains, the parareal algorithm (1.5) will generate a set of values $\tilde{\mathbf{U}}_{n}$ that satisfy $\tilde{\mathbf{U}}_{n}=\mathcal{F}^{\Delta T_{n-1}}\left(\tilde{\mathbf{U}}_{n-1}\right)$. That is, the approximations at the time-points $T_{n}$ will have achieved the accuracy of the propagator $\mathcal{F}$. Nevertheless, 
the main idea of the algorithm is to choose propagators $\mathcal{F}$ and $\mathcal{G}$ in order to achieve this level of accuracy without performing the $N$ accurate resolutions. Therefore, after convergence of the algorithm (1.5) for $i=i_{\text {conv }}$, we shall obtain a solution $\tilde{\mathbf{U}}_{n}^{i_{\text {conv }}}$ with a fine accuracy respect to $\mathbf{U}_{n}$ in (1.3), for which only $i_{\text {conv }} \ll N$ subdomains would have been integrated by propagator $\mathcal{F}$. Thus from an initial coarse approximation (1.4), we might achieve an accurate resolution of problem (1.1) with important savings of computational time.

\subsection{Interpretation of the parareal algorithm}

The parareal algorithm can most naturally be interpreted as a classical deferred correction method in which the initial values $\mathbf{U}_{n}$ of (1.2) are corrected through a feedback mechanism based on both propagators $\mathcal{F}$ and $\mathcal{G}$, as it was presented in [3]. Therefore, for a initial condition given by (1.4), we define the correction $\mathcal{E}^{\Delta T_{n-1}}\left(\tilde{\mathbf{U}}_{n-1}\right)=$ $\mathcal{F}^{\Delta T_{n-1}}\left(\tilde{\mathbf{U}}_{n-1}\right)-\mathcal{G}^{\Delta T_{n-1}}\left(\tilde{\mathbf{U}}_{n-1}\right)$ in order to obtain the new initial conditions for $n=1, \ldots, N-1$ and the new value at $n=N$ :

$$
\tilde{\mathbf{U}}_{n}^{1}=\mathcal{G}^{\Delta T_{n-1}}\left(\tilde{\mathbf{U}}_{n-1}^{1}\right)+\mathcal{E}^{\Delta T_{n-1}}\left(\tilde{\mathbf{U}}_{n-1}^{0}\right) .
$$

Thus, a recursive application leads us to

$$
\tilde{\mathbf{U}}_{0}^{i}=\mathbf{u}^{0}, \quad \tilde{\mathbf{U}}_{n}^{i}=\mathcal{G}^{\Delta T_{n-1}}\left(\tilde{\mathbf{U}}_{n-1}^{i}\right)+\mathcal{E}^{\Delta T_{n-1}}\left(\tilde{\mathbf{U}}_{n-1}^{i-1}\right) \text { for } n=1, \ldots, N
$$

which gives us the same scheme as (1.5).

Nevertheless, based on [7], the time decomposition method (1.2) can be also interpreted as a multiple shooting method for (1.1). In fact, considering $\mathbf{U}=\left(\mathbf{U}_{0}, \ldots, \mathbf{U}_{N}\right)^{T}$ as the unknowns, the system (1.3) can be written as

$$
\mathbf{F}(\mathbf{U})=\left(\begin{array}{c}
\mathbf{U}_{0}-\mathbf{u}^{0} \\
\mathbf{U}_{1}-\phi^{\Delta T_{0}}\left(\mathbf{U}_{0}\right) \\
\vdots \\
\mathbf{U}_{N}-\phi^{\Delta T_{N-1}}\left(\mathbf{U}_{N-1}\right)
\end{array}\right)=\mathbf{0}
$$

where $\mathbf{F}: \mathbb{R}^{M \cdot N+1} \rightarrow \mathbb{R}^{M \cdot N+1}$. In this way, we search the initial values $\mathbf{U}_{n}$ of (1.2) that correspond to the solution of original problem (1.1) through condition (1.3).

Solving this system with Newton's method, leads after a short calculation to

$$
\tilde{\mathbf{U}}_{0}^{i}=\mathbf{u}^{0}, \quad \tilde{\mathbf{U}}_{n}^{i}=\phi^{\Delta T_{n-1}}\left(\tilde{\mathbf{U}}_{n-1}^{i-1}\right)+\frac{\partial \phi^{\Delta T_{n-1}}\left(\tilde{\mathbf{U}}_{n-1}^{i-1}\right)}{\partial \tilde{\mathbf{U}}_{n-1}^{i-1}}\left(\tilde{\mathbf{U}}_{n-1}^{i}-\tilde{\mathbf{U}}_{n-1}^{i-1}\right) \quad \text { for } n=1, \ldots, N
$$

With this formulation, there are many ways to apply the multiple shooting algorithm to solve (1.8) numerically, as it is detailed in [28], and when the approximations are close enough to the solution, the convergence is guaranteed as shown in [7]. However, if we approximate the time subdomain integration in (1.8) by

$$
\phi^{\Delta T_{n-1}}\left(\tilde{\mathbf{U}}_{n-1}^{i-1}\right) \approx \mathcal{F}^{\Delta T_{n-1}}\left(\tilde{\mathbf{U}}_{n-1}^{i-1}\right),
$$

and the Jacobian term by

$$
\frac{\partial \phi^{\Delta T_{n-1}}\left(\tilde{\mathbf{U}}_{n-1}^{i-1}\right)}{\partial \tilde{\mathbf{U}}_{n-1}^{i-1}}\left(\tilde{\mathbf{U}}_{n-1}^{i}-\tilde{\mathbf{U}}_{n-1}^{i-1}\right) \approx \mathcal{G}^{\Delta T_{n-1}}\left(\tilde{\mathbf{U}}_{n-1}^{i}\right)-\mathcal{G}^{\Delta T_{n-1}}\left(\tilde{\mathbf{U}}_{n-1}^{i-1}\right),
$$

then the multiple shooting method (1.8) and the parareal algorithm (1.5) coincide (see [28]).

The parareal algorithm can also be seen as a time-multigrid method as it was entirely proven in [28]. However, because of its clarity and simplicity, in this paper we will adopt the multiple shooting point of view, i.e. a Newton's method approximation, even if the numerical analysis we will perform is practically independent of this choice. In fact, from a practical point of view, all the interpretations are equivalent and the algorithm is mainly implemented as a deferred correction method. 


\subsection{Considerations on the propagation operators}

The parareal algorithm relies on two solvers, the fine and coarse propagators, $\mathcal{F}$ and $\mathcal{G}$. On the one hand, the fine solver produces an accurate approximation of the solution and its choice depends on the desired level of accuracy and on the nature of the problem (multi-scale phenomena, stiffness, large systems). In our particular case of multi-scale reaction waves, the time operator splitting reveals itself as a suitable resolution technique as it was previously discussed in the introduction. Its parallelization is then achieved via the parareal scheme. On the other hand, the coarse solver gives a coarser approximation that needs to be computed in a sequential way, therefore it should be as fast as possible in order to globally guarantee important savings in time consumption. The performance of the parareal algorithm then relies directly on the choice of this solver and an optimal balance must be found between its computational speed and its level of accuracy, i.e. coarser approximations are faster but increase the number of iterations needed.

Let us now set the general mathematical framework in this work; in this context, we recall that a class of multi-scale phenomena can be modeled by general reaction-diffusion systems of type:

$$
\left.\begin{array}{ll}
\partial_{t} \mathbf{u}-\partial_{\mathbf{x}}\left(\mathbf{D}(\mathbf{u}) \partial_{\mathbf{x}} \mathbf{u}\right)=\mathbf{f}(\mathbf{u}), & \mathbf{x} \in \mathbb{R}^{d}, t>0 \\
\mathbf{u}(0, \mathbf{x})=\mathbf{u}_{0}(\mathbf{x}), & \mathbf{x} \in \mathbb{R}^{d}, t=0
\end{array}\right\}
$$

where $\mathbf{f}: \mathbb{R}^{M} \rightarrow \mathbb{R}^{M}$ and $\mathbf{u}: \mathbb{R} \times \mathbb{R}^{d} \rightarrow \mathbb{R}^{M}$ for some integer $d$ and with the diffusion matrix $\mathbf{D}$ (u), which is a tensor of order $d \times d \times M$.

However, in this work we will only consider the simplified case of linear diagonal diffusion, in which case the elements of the diffusion matrix are written as $D_{i_{1} i_{2} i_{3}}(\mathbf{u})=D_{i_{3}} \delta_{i_{1} i_{2}}$, so that the diffusion operator reduces to the heat operator with some scalar diffusion coefficient $D_{i_{3}}$ for component $u_{i_{3}}$ of $\mathbf{u}$. Notice that as it was established in previous studies [13,16,17], on the one hand, a rigorous numerical analysis of this simpler class of reaction-diffusion systems is very useful in order to theoretically characterize the impact of stiffness on the numerical behavior of methods conceived to simulate these phenomena. In particular, the original multi-scale character is not withdrawn. And on the other hand, it shall give us some insights into more complex cases as well as complete convection-reaction-diffusion systems.

Finally, in order to simplify the presentation, we consider a one-dimensional model, taking into account that extension into higher dimensions of $\mathbf{x}$ or $\mathbf{u}$ is straightforward. From these considerations, we infer the following initial value problem:

$$
\left.\begin{array}{ll}
\partial_{t} u-D \partial_{x}^{2} u=k f(u), & x \in \mathbb{R}, t>0, \\
u(0, x)=u_{0}(x), & x \in \mathbb{R}, t=0,
\end{array}\right\}
$$

where $f$ and $u_{0}$ are smooth functions, with real coefficients $D$ and $k$. Furthermore, we denote by $T^{t}\left(u_{0}\right)$ the semiflow associated to (1.10). In what follows, we shall present both the fine and the coarse solvers taken into consideration to solve problem (1.10) by means of the parareal algorithm (1.5).

\subsubsection{Fine solver: time operator splitting}

Let us first introduce the classical decoupling of the diffusion and reaction parts of (1.10). More precisely, we denote $X^{t}\left(u_{0}\right)$ the solution of the diffusion equation:

$$
\partial_{t} u_{D}-D \partial_{x}^{2} u_{D}=0, \quad x \in \mathbb{R}, t>0
$$

with initial data $u_{D}(0, \cdot)=u_{0}(\cdot)$. We also denote by $Y^{t}\left(u_{0}\right)$ the solution of the reaction part where the spatial coordinate $x$ can be considered as a parameter:

$$
\partial_{t} u_{R}=k f\left(u_{R}\right), \quad x \in \mathbb{R}, t>0,
$$

with initial data $u_{R}(0, \cdot)=u_{0}(\cdot)$. 
The two Lie approximation formulae of (1.10) are defined by

$$
L_{1}^{t}\left(u_{0}\right)=X^{t} Y^{t}\left(u_{0}\right), \quad L_{2}^{t}\left(u_{0}\right)=Y^{t} X^{t}\left(u_{0}\right)
$$

and the two Strang approximation formulae of $(1.10)[59,60]$ are defined by

$$
S_{1}^{t}\left(u_{0}\right)=X^{t / 2} Y^{t} X^{t / 2}\left(u_{0}\right), \quad S_{2}^{t}\left(u_{0}\right)=Y^{t / 2} X^{t} Y^{t / 2}\left(u_{0}\right)
$$

It is well known that Lie formulae (1.13) (resp. Strang formulae (1.14)) are an approximation of order 1 (resp. 2) of the exact solution of (1.10). Nevertheless, these classical orders are no longer valid since we consider very stiff reactive or diffusive terms (see [13]). In fact, if the fastest time scales play a leading role in the global physics of the phenomenon, then the composed solution obtained by means of a splitting technique will surely fail to capture the final dynamics of the phenomenon, unless we consider splitting time steps of the same order of such scales.

In the opposite case where these fast scales are not directly related to the physical development of the phenomenon, larger splitting time steps might be considered, but order reductions may then appear due to short-life transients associated to the fast variables. In particular, this is the case for propagating reaction waves. In this context, it has been proven in [13] that better performances are expected while ending the splitting scheme by the time integration of the reaction part (1.12) or in a more general case, the part involving the fastest time scales of the phenomenon (see the numerical application in [17]). In particular, in the case of linear diagonal diffusion problems, no order loss is expected for the $L_{2}^{t}$ and $S_{2}^{t}$ schemes when fast scales are present in the reactive term. Even more, as it was presented and analyzed in [16], the presence of high spatial gradients may also degrade the performance of these methods leading to order reductions coming from space multi-scale phenomena.

Keeping in mind these theoretical studies and considering the various numerical alternatives previously discussed, the time operator splitting remains as the most appropriate resolution scheme for general multi-scale problems and so far, the optimal choice for the fine solver. A complementary consideration is that suitable time integration methods in terms of order and stability must be chosen for each subsystem (1.11) and (1.12), in order to guarantee the accuracy of the estimates established by the corresponding numerical analysis. In fact, in all splitting order estimates, the solutions associated to these systems are supposed to be known exactly or with a sufficient accuracy (see for example $[13,14,36,37]$ ).

\subsubsection{Coarse approximation}

When considering stiff problems of type (1.9) (or (1.10)), the choice of the coarse solver is not an easy task because we must look for fast and stable methods at the same time, considering that these computations will be performed in a sequential environment. In fact, depending on the stiffness of the system, we are almost constrained to choose more expensive but more stable methods (see [35] for more details on integration of stiff ODEs), otherwise, we would not be able to obtain coarser but still valid approximations.

Taking into account these requirements, several strategies might be considered. For instance, we can solve the coupled reaction-diffusion system (1.10) with a dedicated, stable but less accurate solver; which can be achieved by using larger integration time steps or a lower order method respect to the fine solver. Nevertheless, as previously discussed, the important computational requirements of these dedicated methods must be taken into account and thus, this alternative might not be always feasible.

Another technique could be the resolution of (1.10), discretized on a coarser spatial grid. Then, the crucial aspect would be the definition of proper inter-grid operators; that is, the operators allowing data exchange between the coarse and the fine grids. On the other hand, we might also consider the resolution of a reduced model of (1.10) instead, where the fastest scales have been relaxed; and thus, take this solution as the coarse approximation. Nevertheless, the previous knowledge of the fast scales is mandatory for straightforward implementations, and at the same time, we should also conceive an efficient reconstruction procedure of the fast variables not estimated by the coarse solver. 
However, in the context of multi-scale reaction waves, a less accurate splitting operator might be a natural choice, conceived with larger splitting time steps or coarser time integrators of the split subsystems in order to accelerate computations. In particular, in this work we will be mainly focused on the detailed analysis of such splitting solvers as coarse propagators, considering their reputed pertinence on multi-scale problems. In this way, the numerical analysis presented in the next section describes the behavior of the splitting techniques as well as the parareal scheme itself, and shall lead us to further numerical studies and potential improvements of the numerical strategy.

\section{NUMERICAL ANALYSIS OF THE PARAREAL OPERATOR SPLITTING}

In this section, we will first present important results from the literature that explain the behavior of the parareal algorithm and detail its convergence when applied to, first, linear and then, nonlinear problems. Those particularly relevant for our study will be described in details. Complementary information can be found in the indicated references. Then, a comprehensive numerical analysis of the parareal Lie (Strang) operator splitting will be presented in a linear framework that mimics the eventual influence of high spatial gradients for reaction waves.

Throughout all this section, we will maintain the notation established in the previous one.

\subsection{Review of the literature and state of the art}

\subsubsection{The linear case}

We first consider a scalar linear problem of the form:

$$
u^{\prime}(t)=a u, \quad t \in[0, T], \quad u(0)=u^{0} \text { with } a \in \mathbb{C},
$$

the following proposition is taken from the first publication on the parareal algorithm [42].

Proposition 2.1. Let $\Delta T=T / N, T_{n}=n \Delta T$ for $n=0, \ldots, N$. Consider (2.1) with a $\in \mathbb{R}$. Let $\mathcal{F}^{\Delta T}\left(U_{n}\right)$ be the exact solution at $T_{n+1}$ of $(2.1)$ with $u\left(T_{n}\right)=U_{n}$, and let $\mathcal{G}^{\Delta T}\left(U_{n}\right)$ be the corresponding backward Euler approximation with time step $\Delta T$. Then,

$$
\max _{1 \leq n \leq N}\left|u\left(T_{n}\right)-\tilde{U}_{n}^{i}\right| \leq P_{i} \Delta T^{i+1}
$$

Thus, the algorithm converges and behaves in $\Delta T$ like a method of order $i+1$. Nevertheless, we must take into account that this result is only valid for a fixed iteration step $i$, since the constant $P_{i}$ in $(2.2)$ grows with $i$ in the estimate of the proof in [42], i.e. the convergence is verified only for $\Delta T$ sufficiently small. Result (2.2) has been extended to more general and, in particular, higher order time-integration schemes. In fact, it was shown in $[4,5]$ that the parareal is a method of order $p(i+1)$ when a method of order $p$ is used as the coarse propagator.

However, a very important work presented in [28] gives us complementary results that describe the behavior of the algorithm for any $i$ and a fixed $\Delta T$. As a matter of fact, the following corollary was stated and proven.

Corollary 2.2. Let $T<\infty, \Delta T=T / N$, and $n=0, \ldots, N$. Consider (2.1) with a $\in \mathbb{C}$. Let $\mathcal{F}^{\Delta T}\left(U_{n}\right)$ be the exact solution at $T_{n+1}$ of $(2.1)$ with $u\left(T_{n}\right)=U_{n}$, and let $\mathcal{G}^{\Delta T}\left(U_{n}\right)=R(a \Delta T) U_{n}$ be a one-step method in its region of absolute stability. Then, we have the bound

$$
\max _{1 \leq n \leq N}\left|u\left(T_{n}\right)-\tilde{U}_{n}^{i}\right| \leq \frac{\left|\mathrm{e}^{a \Delta T}-R(a \Delta T)\right|^{i}}{i !} \prod_{j=1}^{i}(N-j) \max _{1 \leq n \leq N}\left|u\left(T_{n}\right)-\tilde{U}_{n}^{0}\right| .
$$


If the local truncation error of $\mathcal{G}$ is bounded by $C \Delta T^{p+1}$, with $p>0$ and $C$ a constant, then we have, for $\Delta T$ small enough,

$$
\max _{1 \leq n \leq N}\left|u\left(T_{n}\right)-\tilde{U}_{n}^{i}\right| \leq \frac{(C T)^{i}}{i !} \Delta T^{p i} \max _{1 \leq n \leq N}\left|u\left(T_{n}\right)-\tilde{U}_{n}^{0}\right|
$$

These results give us more precise information regarding constant $P_{i}$ in (2.2). In fact, for finite $T$, the division by $i$ ! in (2.3) and (2.4) shows that the algorithm converges superlinearly. Furthermore, result (2.4) presents the parareal as a method of order $p i$.

A linear convergence result was also demonstrated in [28] for an infinitely long time interval, i.e. $T=\infty$, for problem (2.1). Moreover, similar results were obtained in [28] while investigating the performance of the parareal algorithm on PDEs, more precisely, a diffusion equation and an advection one. In fact, a Fourier transform in space converts these equations into linear systems of ODEs for each Fourier mode, and the convergence is demonstrated based on the previous results with some complementary hypothesis.

\subsubsection{The nonlinear case}

Taking into account the nonlinear system of ODEs (1.1), the superlinear convergence of the parareal algorithm has also been demonstrated in [27]. In fact, considering $\mathcal{F}$ as the exact solution of (1.1), it has been assumed that the difference between the approximate solution given by $\mathcal{G}$ and the exact solution can be expanded for $\Delta T$ small, in the following way:

$$
\mathcal{F}^{\Delta T}(\mathbf{u})-\mathcal{G}^{\Delta T}(\mathbf{u})=c_{p+1}(\mathbf{u}) \Delta T^{p+1}+c_{p+2}(\mathbf{u}) \Delta T^{p+2}+\ldots,
$$

which is possible if the right hand side function $\mathbf{f}$ in (1.1) is smooth enough. Notice that this condition is no other than the series expansion of the local error of a numerical method $\mathcal{G}$ of order $p$ (where $\mathcal{F}$ is the exact solution of (1.1)). Then, as a consequence of (2.5), we might assume that we have the following bound:

$$
\left\|\mathcal{F}^{\Delta T}(\mathbf{u})-\mathcal{G}^{\Delta T}(\mathbf{u})-\left(\mathcal{F}^{\Delta T}(\mathbf{v})-\mathcal{G}^{\Delta T}(\mathbf{v})\right)\right\| \leq C_{1} \Delta T^{p+1}\|\mathbf{u}-\mathbf{v}\|,
$$

as it has been supposed in [27].

Moreover, if $\mathcal{G}$ satisfies the Lipschitz condition:

$$
\left\|\mathcal{G}^{\Delta T}(\mathbf{u})-\mathcal{G}^{\Delta T}(\mathbf{v})\right\| \leq\left(1+C_{2} \Delta T\right)\|\mathbf{u}-\mathbf{v}\|,
$$

the following theorem was stated and proven in [27].

Theorem 2.3. Let $\mathcal{F}^{\Delta T}\left(\mathbf{U}_{n-1}\right)$ be the exact solution on time subdomain $I_{n-1}$, and let $\mathcal{G}^{\Delta T}\left(\mathbf{U}_{n-1}\right)$ be an approximate solution with local truncation error bounded by $C_{3} \Delta T^{p+1}$, and satisfying $(2.5)$, where the $c_{j}$, $j=p+1, p+2, \ldots$ are continuously differentiable, and assume that $\mathcal{G}$ satisfies the Lipschitz condition (2.7). Then, at iteration $i$ of the parareal algorithm (1.5), we have the bound

$$
\begin{aligned}
\left\|\mathbf{u}\left(T_{n}\right)-\tilde{\mathbf{U}}_{n}^{i}\right\| & \leq \frac{C_{3}}{C_{1}} \frac{\left(C_{1} \Delta T^{p+1}\right)^{i+1}}{(i+1) !}\left(1+C_{2} \Delta T\right)^{n-i-1} \prod_{j=0}^{i}(n-j), \\
& \leq \frac{C_{3}}{C_{1}} \frac{\left(C_{1} T_{n}\right)^{i+1}}{(i+1) !} \mathrm{e}^{C_{2}\left(T_{n}-T_{i+1}\right)} \Delta T^{p(i+1)}
\end{aligned}
$$

Finally, we see that the parareal algorithm also converges superlinearly with respect to $i$ and behaves as a method of order $p(i+1)$ for a nonlinear problem and a finite $T$. Hence, result $(2.8)$ does not contradict the previous ones but extends them to the nonlinear case. Nevertheless, in a practical application, a more complete knowledge of the bound (2.8) might be necessary in order to fully describe the behavior of the algorithm. More precisely, constants $C_{1}, C_{2}$ and $C_{3}$ in (2.8) (as well as $C$ into (2.4) for a linear application) should be properly estimated. This is one of the purposes of the present work in the case of an operator splitting strategy and the corresponding analysis will be presented in the next section. 


\subsection{Convergence analysis of the parareal operator splitting}

In order to carry on our study, we will first present the mathematical problem that we want to solve. The link with multi-scale reaction waves will be then established.

\subsubsection{Mathematical framework}

We consider the initial-value problem:

$$
\left.\begin{array}{ll}
\partial_{t} u-D \partial_{x}^{2} u+k V(x) u=0 & x \in \mathbb{R}, t>0, \\
u(x, 0)=u_{0}(x) & x \in \mathbb{R}, t=0
\end{array}\right\}
$$

where $V$ is a bounded function from $\mathbb{R}$ to $\mathbb{R}$ of class $\mathrm{C}^{\infty}$ with all bounded derivatives and, $D$ and $k$ are some real positive coefficients such that $D k=1$. Moreover, $u$ represents a dimensionless specie concentration.

For this study, we consider problem (2.9) as coming from the associated linearized system of (1.10) around $u_{0}$ after some simple change of variables. In this context, $V$ is no other than the Jacobian $f^{\prime}\left(u_{0}\right)=\partial f\left(u_{0}\right) / \partial u$ in (1.10). The diffusion and reaction coefficients, $D$ and $k$ in (1.10) and (2.9), might be seen as scale coefficients of time and space. In fact, in the context of reaction waves, we can obtain a dimensionless form of system (1.10) considering a dimensionless time $\tau$ and a dimensionless space $r$ with

$$
\tau=k t \quad \text { and } \quad r=(k / D)^{1 / 2} x
$$

See [37] for details on how reaction systems of ODEs can be built from a reaction scheme, and [33] for details on analysis of traveling reaction waves.

Then, without loss of generality, a dimensionless analysis of a traveling wave, as shown in [33], may allow us to establish the steady state of a reaction wave. Therefore, taking into account the scale coefficients, the dimensionless steady velocity of the wavefront given by the expression $\mathrm{d} r / \mathrm{d} \tau$ establishes that

$$
\frac{\mathrm{d} x}{\mathrm{~d} t} \propto(D k)^{1 / 2}
$$

while coming back to the initial representation of time $t$ and space $x$. Moreover, in a general way, the sharpness of the wave profile is measured by

$$
\left.\frac{\mathrm{d} u}{\mathrm{~d} x}\right|_{\max } \propto(k / D)^{1 / 2} .
$$

Note that condition $D k=1$ implies constant velocity for all $k=1 / D$; however, greater $k$ (or smaller $D$ ) implies higher spatial gradients, and thus, stiffer configurations.

In the context of reaction waves, we have usually wavefronts of steep spatial gradient propagating with a steady constant speed. Therefore, we are interested in studying this kind of stiff configurations and their impact on a time parallelization algorithm such as the parareal scheme. In particular, the latter must be coupled with an appropriate stiff solver such as the operator splitting, considered in this work. Hence, in order to conduct a convergence analysis of the parareal scheme, we are first constrained to obtain some bounds (similar to those given by (2.5), (2.6) and (2.7)) drawn out of a more precise analysis of the operator splitting itself, applied to problem (2.9). This is the main goal of the following section.

\subsubsection{Analysis of the operator splitting}

For problem (2.9), we consider the linear operator $b$ corresponding to the multiplication by $k V$ and the operator $a=-D \partial_{x}^{2}$ (minus $D$ times the second partial derivative with respect to $x$ in one dimension). Hence, their commutator is given by

$$
\partial_{a} b=[a, b]=-\left(\partial_{x}^{2} V\right)-2\left(\partial_{x} V\right) \partial_{x}
$$


considering that $D k=1$. We first recall the following result which is proven in [14] and gives an exact representation of the difference between the exponential of $a+b$, i.e. the exact solution of (2.9), and its Lie (resp. Strang) approximation denoted by $L$ (resp. $S$ ).

Theorem 2.4. The following identities hold

$$
\begin{aligned}
L(t)= & \mathrm{e}^{-t a} \mathrm{e}^{-t b}=\mathrm{e}^{-t(a+b)}+\int_{0}^{t} \int_{0}^{s} \mathrm{e}^{-(t-s)(a+b)} \mathrm{e}^{-(s-r) a}\left(\partial_{a} b\right) \mathrm{e}^{-r a} \mathrm{e}^{-s b} \mathrm{~d} r \mathrm{~d} s, \\
S(t)= & \mathrm{e}^{-t b / 2} \mathrm{e}^{-t a} \mathrm{e}^{-t b / 2}=\mathrm{e}^{-t(a+b)}+\frac{1}{4} \int_{0}^{t} \int_{0}^{s}(s-r) \mathrm{e}^{-(t-s)(a+b)} \mathrm{e}^{-(s-r) b / 2}\left(\partial_{b}^{2} a\right) \mathrm{e}^{-r b / 2} \mathrm{e}^{-s a} \mathrm{e}^{-s b / 2} \mathrm{~d} r \mathrm{~d} s \\
& -\frac{1}{2} \int_{0}^{t} \int_{0}^{s}(s-r) \mathrm{e}^{-(t-s)(a+b)} \mathrm{e}^{-s b / 2} \mathrm{e}^{-r a}\left(\partial_{a}^{2} b\right) \mathrm{e}^{-(s-r) a} \mathrm{e}^{-s b / 2} \mathrm{~d} r \mathrm{~d} s .
\end{aligned}
$$

In particular, for problem (2.9) we have

$$
\begin{aligned}
\partial_{a}^{2} b & =[a,[a, b]]=D\left(\partial_{x}^{4} V\right)+4 D\left(\partial_{x}^{3} V\right) \partial_{x}+4 D\left(\partial_{x}^{2} V\right) \partial_{x}^{2}, \\
\partial_{b}^{2} a & =[b,[b, a]]=-2 k\left(\partial_{x} V\right)^{2} .
\end{aligned}
$$

Identities (2.13) and (2.14) are also valid for general nonlinear operators (or vector fields) $a$ and $b$, in which case the commutators (2.12), (2.15) and (2.16) are computed as the Lie derivatives of the Lie bracket of the vector fields (see [37] and the complete analysis conducted in $[17,43]$ ).

Nevertheless, even if (2.13) and (2.14) give us the exact representation of the local error of a Lie or Strang splitting approximation, we are more interested in defining some bounds on these local errors. More precisely, we will see in the next section that the key point of the proof of the convergence estimates for the parareal algorithm is the use of $\mathrm{L}^{2}$ to $\mathrm{L}^{2}$ estimates of the errors.

Therefore, considering the exact solution of problem (2.9), a simple computation shows that for $t \geq 0$,

$$
\left\|\mathrm{e}^{t\left(D \partial_{x}^{2}-k V\right)}\right\|_{\mathcal{L}\left(\mathrm{L}^{2}, \mathrm{~L}^{2}\right)} \leq \mathrm{e}^{k\|V\|_{\infty} t}
$$

Moreover, with the exact representation of the local errors (2.13) and (2.14), and denoting by

$$
\mathrm{E}_{L}(t)=\mathrm{e}^{t\left(D \partial_{x}^{2}-k V\right)}-L(t) \text { and } \quad \mathrm{E}_{S}(t)=\mathrm{e}^{t\left(D \partial_{x}^{2}-k V\right)}-S(t),
$$

it is straightforward to obtain the following bounds.

Lemma 2.5 (first estimate of the splitting local error). Consider problem (2.9) and $u_{0} \in \mathrm{H}^{2}(\mathbb{R})$, for $t \geq 0$ we have the following error bounds

$$
\left\|E_{L}(t) u_{0}\right\|_{L^{2}} \leq\left(\frac{t^{2}}{2}\left\|\partial_{x}^{2} V\right\|_{\infty}+\frac{2 t^{3}}{3} k\left\|\partial_{x} V\right\|_{\infty}^{2}\right) \mathrm{e}^{k\|V\|_{\infty} t}\left\|u_{0}\right\|_{\mathrm{L}^{2}}+t^{2}\left\|\partial_{x} V\right\|_{\infty} \mathrm{e}^{k\|V\|_{\infty} t}\left\|\partial_{x} u_{0}\right\|_{\mathrm{L}^{2}},
$$

and

$$
\begin{aligned}
\left\|E_{S}(t) u_{0}\right\|_{\mathrm{L}^{2}} \leq & \left(\frac{t^{3}}{12}\left(k\left\|\partial_{x} V\right\|_{\infty}^{2}+D\left\|\partial_{x}^{4} V\right\|_{\infty}\right)+\frac{t^{4}}{8}\left(\left\|\partial_{x}^{3} V\right\|_{\infty}\left\|\partial_{x} V\right\|_{\infty}+\left\|\partial_{x}^{2} V\right\|_{\infty}^{2}\right)\right. \\
& \left.+\frac{t^{5}}{20} k\left\|\partial_{x}^{2} V\right\|_{\infty}\left\|\partial_{x} V\right\|_{\infty}^{2}\right) \mathrm{e}^{k\|V\|_{\infty} t}\left\|u_{0}\right\|_{\mathrm{L}^{2}}+\left(\frac{t^{3}}{3} D\left\|\partial_{x}^{3} V\right\|_{\infty}\right. \\
& \left.+\frac{t^{4}}{4}\left\|\partial_{x}^{2} V\right\|_{\infty}\left\|\partial_{x} V\right\|_{\infty}\right) \mathrm{e}^{k\|V\|_{\infty} t}\left\|\partial_{x} u_{0}\right\|_{\mathrm{L}^{2}}+\frac{t^{3}}{3} D\left\|\partial_{x}^{2} V\right\|_{\infty} \mathrm{e}^{k\|V\|_{\infty} t}\left\|\partial_{x}^{2} u_{0}\right\|_{\mathrm{L}^{2}}
\end{aligned}
$$


Proof. Using (2.13), we have in $\mathrm{L}^{2}(\mathbb{R})$,

$$
\left\|\mathrm{e}^{t\left(D \partial_{x}^{2}-k V\right)} u_{0}-\mathrm{e}^{t D \partial_{x}^{2}} \mathrm{e}^{-t k V} u_{0}\right\|_{\mathrm{L}^{2}} \leq \int_{0}^{t} \int_{0}^{s}\left\|\mathrm{e}^{(t-s)\left(D \partial_{x}^{2}-k V\right)} \mathrm{e}^{(s-r) D \partial_{x}^{2}} \partial_{a} b \mathrm{e}^{r D \partial_{x}^{2}} \mathrm{e}^{-s k V} u_{0}\right\|_{\mathrm{L}^{2}} \mathrm{~d} r \mathrm{~d} s .
$$

And with (2.17) we have

$$
\left\|\mathrm{e}^{t\left(D \partial_{x}^{2}-k V\right)} u_{0}-\mathrm{e}^{t D \partial_{x}^{2}} \mathrm{e}^{-t k V} u_{0}\right\|_{\mathrm{L}^{2}} \leq \int_{0}^{t} \int_{0}^{s} \mathrm{e}^{(t-s) k\|V\|_{\infty}}\left\|\partial_{a} b \mathrm{e}^{r D \partial_{x}^{2}} \mathrm{e}^{-s k V} u_{0}\right\|_{\mathrm{L}^{2}} \mathrm{~d} r \mathrm{~d} s .
$$

Since

$$
\begin{aligned}
\partial_{a} b \mathrm{e}^{r D \partial_{x}^{2}} \mathrm{e}^{-s k V} u_{0}= & -\left(\partial_{x}^{2} V\right) \mathrm{e}^{r D \partial_{x}^{2}} \mathrm{e}^{-s k V} u_{0}-2\left(\partial_{x} V\right) \partial_{x}\left(\mathrm{e}^{r D \partial_{x}^{2}} \mathrm{e}^{-s k V} u_{0}\right), \\
= & -\left(\partial_{x}^{2} V\right) \mathrm{e}^{r D \partial_{x}^{2}} \mathrm{e}^{-s k V} u_{0}-2\left(\partial_{x} V\right) \mathrm{e}^{r D \partial_{x}^{2}} \partial_{x}\left(\mathrm{e}^{-s k V} u_{0}\right), \\
= & -\left(\partial_{x}^{2} V\right) \mathrm{e}^{r D \partial_{x}^{2}} \mathrm{e}^{-s k V} u_{0}-2\left(\partial_{x} V\right) \mathrm{e}^{r D \partial_{x}^{2}} \mathrm{e}^{-s k V} \partial_{x} u_{0} \\
& +2\left(\partial_{x} V\right) \mathrm{e}^{r D \partial_{x}^{2}} s k\left(\partial_{x} V\right) \mathrm{e}^{-s k V} u_{0},
\end{aligned}
$$

we obtain (2.18), integrating (2.20) and knowing that $\left\|\mathrm{e}^{-s k V}\right\|_{\mathcal{L}\left(\mathrm{L}^{2}, \mathrm{~L}^{2}\right)} \leq \mathrm{e}^{s k\|V\|_{\infty}}$.

Performing the same computations, we obtain (2.19) from (2.14), (2.15) and (2.16).

However, we recall the fact that we are specifically searching for $\mathrm{L}^{2}$ to $\mathrm{L}^{2}$ error estimates in order to conduct the convergence analysis of the parareal scheme. Hence, thanks to the regularizing effect of the Laplacian, we have for all $u_{0}$ in $\mathrm{L}^{2}$ and for $t>0$,

$$
\left\|\partial_{x} \mathrm{e}^{t D \partial_{x}^{2}} u_{0}\right\|_{\mathrm{L}^{2}} \leq \frac{1}{\sqrt{2 e D t}}\left\|u_{0}\right\|_{\mathrm{L}^{2}}
$$

and then, we can conduct further calculations.

Lemma 2.6 (second estimate of the splitting local error). Consider problem (2.9), for $t>0$ we have the following error bounds

$$
\left\|E_{L}(t) u_{0}\right\|_{\mathrm{L}^{2}} \leq\left(\frac{8}{3} t \sqrt{t}\left(\frac{\left\|\partial_{x} V\right\|_{\infty}}{\sqrt{2 e D}}\right)+\frac{t^{2}}{2}\left\|\partial_{x}^{2} V\right\|_{\infty}\right) \mathrm{e}^{k\|V\|_{\infty} t}\left\|u_{0}\right\|_{\mathrm{L}^{2}},
$$

and

$$
\left\|E_{S}(t) u_{0}\right\|_{\mathrm{L}^{2}} \leq\left(\frac{t^{2}}{4}\left(\frac{\pi}{e}\left\|\partial_{x}^{2} V\right\|_{\infty}\right)+\frac{t^{3}}{12}\left(k\left\|\partial_{x} V\right\|_{\infty}^{2}+D\left\|\partial_{x}^{4} V\right\|_{\infty}\right)\right) \mathrm{e}^{k\|V\|_{\infty} t}\left\|u_{0}\right\|_{\mathrm{L}^{2}} .
$$

Proof. We take into account that

$$
\partial_{a} b \mathrm{e}^{r D \partial_{x}^{2}} \mathrm{e}^{-s k V} u_{0}=-\left(\partial_{x}^{2} V\right) \mathrm{e}^{r D \partial_{x}^{2}} \mathrm{e}^{-s k V} u_{0}-2\left(\partial_{x} V\right) \partial_{x}\left(\mathrm{e}^{r D \partial_{x}^{2}} \mathrm{e}^{-s k V} u_{0}\right) .
$$

Coming back to (2.20), the regularizing effect of the Laplacian (2.21) yields

$$
\begin{aligned}
\left\|\mathrm{e}^{t\left(D \partial_{x}^{2}-k V\right)} u_{0}-\mathrm{e}^{t D \partial_{x}^{2}} \mathrm{e}^{-t k V} u_{0}\right\|_{\mathrm{L}^{2}} & \leq \int_{0}^{t} \int_{0}^{s} \mathrm{e}^{(t-s) k\|V\|_{\infty}}\left\|\partial_{a} b \mathrm{e}^{r D \partial_{x}^{2}} \mathrm{e}^{-s k V} u_{0}\right\|_{\mathrm{L}^{2}} \mathrm{~d} r \mathrm{~d} s \\
& \leq \int_{0}^{t} \int_{0}^{s} \mathrm{e}^{(t-s) k\|V\|_{\infty}}\left(\left(\frac{2\left\|\partial_{x} V\right\|_{\infty}}{\sqrt{2 e D r}}\right)+\left\|\partial_{x}^{2} V\right\|_{\infty}\right)\left\|\mathrm{e}^{-s k V} u_{0}\right\|_{\mathrm{L}^{2}} \mathrm{~d} r \mathrm{~d} s \\
& \leq\left(\frac{8}{3} t \sqrt{t}\left(\frac{\left\|\partial_{x} V\right\|_{\infty}}{\sqrt{2 e D}}\right)+\frac{t^{2}}{2}\left\|\partial_{x}^{2} V\right\|_{\infty}\right) \mathrm{e}^{k\|V\|_{\infty} t}\left\|u_{0}\right\|_{\mathrm{L}^{2}} .
\end{aligned}
$$


Moreover, taking into account that

$$
\partial_{a}^{2} b=D\left(\partial_{x}^{4} V\right)+4 D \partial_{x}\left(\left(\partial_{x}^{2} V\right) \partial_{x}\right)
$$

and if we consider

$$
\begin{aligned}
\mathrm{e}^{r D \partial_{x}^{2}} \partial_{a}^{2} b \mathrm{e}^{(s-r) D \partial_{x}^{2}} \mathrm{e}^{-s k V / 2} u_{0} & =D \mathrm{e}^{r D \partial_{x}^{2}}\left(\partial_{x}^{4} V\right) \mathrm{e}^{(s-r) D \partial_{x}^{2}} \mathrm{e}^{-s k V / 2} u_{0}+4 D \mathrm{e}^{r D \partial_{x}^{2}} \partial_{x}\left(\left(\partial_{x}^{2} V\right) \partial_{x}\right) \mathrm{e}^{(s-r) D \partial_{x}^{2}} \mathrm{e}^{-s k V / 2} u_{0} \\
& =D \mathrm{e}^{r D \partial_{x}^{2}}\left(\partial_{x}^{4} V\right) \mathrm{e}^{(s-r) D \partial_{x}^{2}} \mathrm{e}^{-s k V / 2} u_{0}+4 D \partial_{x}\left(\mathrm{e}^{r D \partial_{x}^{2}}\left(\partial_{x}^{2} V\right) \partial_{x} \mathrm{e}^{(s-r) D \partial_{x}^{2}} \mathrm{e}^{-s k V / 2} u_{0}\right)
\end{aligned}
$$

we obtain with $(2.21)$,

$$
\begin{aligned}
\left\|\mathrm{e}^{r D \partial_{x}^{2}} \partial_{a}^{2} b \mathrm{e}^{(s-r) D \partial_{x}^{2}} \mathrm{e}^{-s k V / 2} u_{0}\right\|_{\mathrm{L}^{2}} & \leq D\left\|\partial_{x}^{4} V\right\|_{\infty}\left\|\mathrm{e}^{-s k V / 2} u_{0}\right\|_{\mathrm{L}^{2}}+\frac{4 D}{\sqrt{2 e D r}}\left\|\partial_{x}^{2} V\right\|_{\infty}\left\|\partial_{x} \mathrm{e}^{(s-r) D \partial_{x}^{2}} \mathrm{e}^{-s k V / 2} u_{0}\right\|_{\mathrm{L}^{2}} \\
& \leq D\left\|\partial_{x}^{4} V\right\|_{\infty}\left\|\mathrm{e}^{-s k V / 2} u_{0}\right\|_{\mathrm{L}^{2}}+\frac{2\left\|\partial_{x}^{2} V\right\|_{\infty}}{e \sqrt{r(s-r)}}\left\|\mathrm{e}^{-s k V / 2} u_{0}\right\|_{\mathrm{L}^{2}} \\
& \leq\left(D\left\|\partial_{x}^{4} V\right\|_{\infty}+\frac{2\left\|\partial_{x}^{2} V\right\|_{\infty}}{e \sqrt{r(s-r)}}\right) \mathrm{e}^{s k\|V\|_{\infty} / 2}\left\|u_{0}\right\|_{\mathrm{L}^{2}}
\end{aligned}
$$

Taking this into (2.14) and integrating yield (2.23).

The main advantage of these local error bounds is that all terms in estimates (2.18), (2.19), (2.22) and (2.23) are known. No truncated terms in $t$ are present indeed, since we have considered an exact representation of errors (2.13) and (2.14). Furthermore, first and second estimates of the splitting local error agree perfectly with those found in the literature (see [16]) for the case of PDEs with high spatial gradients. In particular, a more general and complete study of the Strang method can be found in [14].

Notice that as it was stated in [16], an order reduction might appear in the local error. Thus, if we consider $k=D=1$ and expand $\mathrm{e}^{\|V\|_{\infty} t}$, we infer from the previous results that for $t>0$,

$$
\left\|\mathrm{E}_{L}(t) u_{0}\right\|_{\mathrm{L}^{2}} \propto \max \left(\left\|\partial_{x} u_{0}\right\|_{\mathrm{L}^{2}} t^{2},\left\|u_{0}\right\|_{\mathrm{L}^{2}} t^{1.5}\right)
$$

and

$$
\left\|\mathrm{E}_{S}(t) u_{0}\right\|_{\mathrm{L}^{2}} \propto \max \left(\left\|\partial_{x} u_{0}\right\|_{\mathrm{L}^{2}} t^{3},\left\|u_{0}\right\|_{\mathrm{L}^{2}} t^{2}\right)
$$

These estimates describe the behavior of the local errors; the first terms are more relevant when $t$ is small and the second ones when $t$ is not small enough and $\left\|\partial_{x} u_{0}\right\|_{\mathrm{L}^{2}}$ is very high. More precisely there exists an explicit constant $\theta>0$ such that for $t \leq \theta,\left\|\mathrm{E}_{L}(t) u_{0}\right\|_{\mathrm{L}^{2}}$ (resp. $\left\|\mathrm{E}_{S}(t) u_{0}\right\|_{\mathrm{L}^{2}}$ ) behaves like $t^{2}$ (resp. $t^{3}$ ) and for $t \geq \theta$, $\left\|\mathrm{E}_{L}(t) u_{0}\right\|_{\mathrm{L}^{2}}$ (resp. $\left.\left\|\mathrm{E}_{S}(t) u_{0}\right\|_{\mathrm{L}^{2}}\right)$ behaves like $t^{1.5}$ (resp. $t^{2}$ ).

These local error estimates are valid for general linear problems of type (2.9). However, in the context of propagating wavefronts, since the $\mathrm{L}^{2}$-norm of $\partial_{x} u_{0}$ is normally very high, it is especially relevant to obtain alternative error estimates which do not involve the derivative of the initial condition. In our particular case, the $\mathrm{L}^{2}$ to $\mathrm{L}^{2}$ error estimates established in Lemma 2.6 allow us to obtain the following bounds which will be necessary for the convergence analysis of the parareal operator splitting scheme.

Lemma 2.7. Consider problem (2.9) with initial conditions $u_{0}$ and $v_{0}$, then for a fixed $t>0$, there exist $\kappa_{L}, \kappa_{S}, C_{L}, C_{S} \in \mathbb{R}^{+}$such that we have the following bounds

$$
\begin{aligned}
\left\|L(t) u_{0}-L(t) v_{0}\right\|_{\mathrm{L}^{2}} & \leq \mathrm{e}^{k\|V\|_{\infty} t}\left\|u_{0}-v_{0}\right\|_{\mathrm{L}^{2}} \\
\left\|E_{L}(t) u_{0}-E_{L}(t) v_{0}\right\|_{\mathrm{L}^{2}} & \leq \kappa_{L} C_{L} t \sqrt{t} \mathrm{e}^{k\|V\|_{\infty} t}\left\|u_{0}-v_{0}\right\|_{\mathrm{L}^{2}}
\end{aligned}
$$


and

$$
\begin{aligned}
\left\|S(t) u_{0}-S(t) v_{0}\right\|_{\mathrm{L}^{2}} & \leq \mathrm{e}^{k\|V\|_{\infty} t}\left\|u_{0}-v_{0}\right\|_{\mathrm{L}^{2}}, \\
\left\|E_{S}(t) u_{0}-E_{S}(t) v_{0}\right\|_{\mathrm{L}^{2}} & \leq \kappa_{S} C_{S} t^{2} \mathrm{e}^{k\|V\| \infty t}\left\|u_{0}-v_{0}\right\|_{\mathrm{L}^{2}} .
\end{aligned}
$$

Proof. Simple calculation of $\left\|\mathrm{e}^{t D \partial_{x}^{2}} \mathrm{e}^{-t k V}\left(u_{0}-v_{0}\right)\right\|_{\mathrm{L}^{2}}$ yields $(2.24)$, as well as $\left\|\mathrm{e}^{-t k V / 2} \mathrm{e}^{t D \partial_{x}^{2}} \mathrm{e}^{-t k V / 2}\left(u_{0}-v_{0}\right)\right\|_{\mathrm{L}^{2}}$ yields (2.26). Besides, estimates (2.25) and (2.27) come from the bounds (2.22) and (2.23) applied to $\left(u_{0}-v_{0}\right)$ with

$$
\begin{aligned}
C_{L} & =\max \left(\frac{8\left\|\partial_{x} V\right\|_{\infty}}{3 \sqrt{2 e D}}, \frac{\left\|\partial_{x}^{2} V\right\|_{\infty}}{2}\right), \\
C_{S} & =\max \left(\frac{\pi\left\|\partial_{x}^{2} V\right\|_{\infty}}{4 e}, \frac{k\left\|\partial_{x} V\right\|_{\infty}^{2}}{12}, \frac{D\left\|\partial_{x}^{4} V\right\|_{\infty}}{12}\right), \\
\kappa_{L} & \geq 1+\sqrt{t} \text { and } \kappa_{S} \geq 1+t .
\end{aligned}
$$

Notice that bounds (2.25) (resp. (2.27)) and (2.24) (resp. (2.26)) correspond to estimates (2.6) and (2.7) respectively in [27], i.e. the difference of local errors for different initial conditions and the Lipschitz condition related to the Lie (resp. Strang) approximation (we can even consider some $C \in \mathbb{R}^{+}$such that e $\|V\|_{\infty} t \leq$ $(1+C t)$ for $t \in(0,1)$ into the different estimates). Moreover, series expansion (2.5) can be associated to $(2.22)$ (resp. (2.23)). In this way, we have completely characterized the constants $C_{1}, C_{2}$ and $C_{3}$ appearing into classical bound (2.8) and a more detailed analysis of the algorithm can be performed.

The choice of the second estimate of the splitting local errors is justified by the fact that reaction waves phenomena involve usually wavefronts of high spatial gradients and that there is no such practical interest in utilizing very small time steps in order to simulate them. Besides, from a mathematical point of view, this allows us to obtain the necessary Lipschitz relations from $\mathrm{L}^{2}$ to $\mathrm{L}^{2}$ spaces.

\subsubsection{Parareal convergence analysis}

The following theorem gives us the convergence rate of the parareal operator splitting algorithm. Its demonstration is based on the preliminary lemmas stated before and on the convergence analysis developed by [27]. For reasons of simplicity, we assume that all the time subdomains are of the same size, i.e. $\Delta T:=\frac{T}{N}$, and $T_{n}=n \Delta T$ for $n=0,1, \ldots, N$.

Theorem 2.8. Let $\mathcal{F}^{\Delta T}\left(U_{n-1}\right)$ be the exact solution of problem (2.9) on time subdomain $I_{n-1}$. If $\mathcal{G}^{\Delta T}\left(U_{n-1}\right)$ is the Lie approximate solution with local error bounded by (2.22) satisfying (2.24) and (2.25), then at iteration $i$ of the parareal algorithm (1.5), there exist some finite $\kappa_{L}, \kappa_{S}, C_{L}, C_{S}, \Lambda_{T_{n}} \in \mathbb{R}^{+}$such that we have the bound

$$
\left\|u\left(T_{n}\right)-\tilde{U}_{n}^{i}\right\|_{\mathrm{L}^{2}} \leq \Lambda_{T_{n}} \frac{\left(\kappa_{L} C_{L} T_{n}\right)^{i+1}}{(i+1) !} \Delta T^{(i+1) / 2}
$$

with $\Lambda_{T_{n}}=\left\|u_{0}\right\|_{\mathrm{L}^{2}} \mathrm{e}^{k\|V\|_{\infty} T_{n}}$. Otherwise, if $\mathcal{G}^{\Delta T}\left(U_{n-1}\right)$ is the Strang approximate solution with local error bounded by (2.23) satisfying (2.26) and (2.27), then we have the bound

$$
\left\|u\left(T_{n}\right)-\tilde{U}_{n}^{i}\right\|_{\mathrm{L}^{2}} \leq \Lambda_{T_{n}} \frac{\left(\kappa_{S} C_{S} T_{n}\right)^{i+1}}{(i+1) !} \Delta T^{(i+1)} .
$$

Proof. The proof is similar to the one conducted in [27]. From the parareal algorithm (1.5), considering that $\mathcal{F}$ is the exact solution of (2.9) and adding and subtracting $\mathcal{G}^{\Delta T}\left(u\left(T_{n-1}\right)\right)$, we obtain

$$
u\left(T_{n}\right)-\tilde{U}_{n}^{i+1}=\mathcal{F}^{\Delta T}\left(u\left(T_{n-1}\right)\right)-\mathcal{G}^{\Delta T}\left(u\left(T_{n-1}\right)\right)-\left(\mathcal{F}^{\Delta T}\left(\tilde{U}_{n-1}^{i}\right)-\mathcal{G}^{\Delta T}\left(\tilde{U}_{n-1}^{i}\right)\right)+\mathcal{G}^{\Delta T}\left(u\left(T_{n-1}\right)\right)-\mathcal{G}^{\Delta T}\left(\tilde{U}_{n-1}^{i+1}\right) .
$$


Hence, taking norms and considering (2.25) (or (2.27)) and (2.24) (or (2.26)), there exist some $\alpha$ and $\beta$ such that

$$
\left\|u\left(T_{n}\right)-\tilde{U}_{n}^{i+1}\right\|_{\mathrm{L}^{2}} \leq \alpha\left\|u\left(T_{n-1}\right)-\tilde{U}_{n-1}^{i}\right\|_{\mathrm{L}^{2}}+\beta\left\|u\left(T_{n-1}\right)-\tilde{U}_{n-1}^{i+1}\right\|_{\mathrm{L}^{2}} .
$$

The classical convergence analysis lead us to study the recurrence relation

$$
\mathrm{e}_{n}^{i+1}=\alpha \mathrm{e}_{n-1}^{i}+\beta \mathrm{e}_{n-1}^{i+1}, \quad \mathrm{e}_{n}^{0}=\gamma+\beta \mathrm{e}_{n-1}^{0},
$$

where $\mathrm{e}_{n}^{i}$ is an upper bound on $\left\|u\left(T_{n}\right)-\tilde{U}_{n}^{i}\right\|_{\mathrm{L}^{2}}$, the global error of the parareal scheme at $T_{n}$ considering the exact solution $u(t)$. After induction and using the binomial series expansion, we obtain the bound [27]

$$
\mathrm{e}_{n}^{i} \leq \gamma \alpha^{i} \beta^{n-i-1}\left(\begin{array}{c}
n \\
i+1
\end{array}\right)
$$

If $\mathcal{G}$ is the Lie approximate solution,

$$
\alpha=\kappa_{L} C_{L} \Delta T \sqrt{\Delta T} \mathrm{e}^{k\|V\|_{\infty} \Delta T}, \quad \beta=\mathrm{e}^{k\|V\|_{\infty} \Delta T} \quad \text { and } \quad \gamma=\kappa_{L} C_{L} \Delta T \sqrt{\Delta T} \mathrm{e}^{k\|V\|_{\infty} \Delta T}\left\|u_{0}\right\|_{\mathrm{L}^{2}},
$$

then,

$$
\begin{aligned}
\left\|u\left(T_{n}\right)-\tilde{U}_{n}^{i}\right\|_{\mathrm{L}^{2}} & \leq\left\|u_{0}\right\|_{\mathrm{L}^{2}} \frac{\left(\kappa_{L} C_{L} \Delta T \sqrt{\Delta T} \mathrm{e}^{k\|V\|_{\infty} \Delta T}\right)^{i+1}}{(i+1) !}\left(\mathrm{e}^{k\|V\|_{\infty} \Delta T}\right)^{n-i-1} \prod_{j=0}^{i}(n-j), \\
& \leq\left\|u_{0}\right\|_{\mathrm{L}^{2}}\left(\mathrm{e}^{k\|V\|_{\infty} \Delta T}\right)^{n} \frac{\left(\kappa_{L} C_{L} n \Delta T \sqrt{\Delta T}\right)^{i+1}}{(i+1) !}, \\
& \leq\left\|u_{0}\right\|_{\mathrm{L}^{2}} \mathrm{e}^{k\|V\|_{\infty} T_{n}} \frac{\left(\kappa_{L} C_{L} T_{n}\right)^{i+1}}{(i+1) !} \Delta T^{(i+1) / 2} .
\end{aligned}
$$

If $\mathcal{G}$ is the Strang approximate solution, we perform the same process to obtain (2.32) with

$$
\alpha=\kappa_{S} C_{S} \Delta T^{2} \mathrm{e}^{k\|V\|_{\infty} \Delta T}, \quad \beta=\mathrm{e}^{k\|V\|_{\infty} \Delta T} \quad \text { and } \quad \gamma=\kappa_{S} C_{S} \Delta T^{2} \mathrm{e}^{k\|V\|_{\infty} \Delta T}\left\|u_{0}\right\|_{\mathrm{L}^{2}} .
$$

These convergence results show that the parareal algorithm converges superlinearly with respect to $i$ and behaves as a method of order $p(i+1)$ for a problem of type (2.9) and a finite $T$. Notice that this time $p=1 / 2$ for the Lie formula instead of classical global order 1 and $p=1$ for Strang instead of 2, according to the order reduction previously discussed and proven in [16]. Thus, results (2.31) and (2.32) do not contradict the classical results from the literature but complement them, giving new and more detailed insights to the performance of the algorithm. In fact, all the terms into bounds (2.31) and (2.32) are known or can be calculated for a general problem of type (2.9), even more, constants $C_{L}, C_{S}, \kappa_{L}$ and $\kappa_{S}$ have been established in the proof of Lemma 2.7. This means that more precise estimates can be obtained for a parareal operator splitting strategy.

\subsection{Parareal operator splitting for reaction waves}

Theorem 2.8 gives us the convergence rate while applying a parareal operator splitting strategy on a general problem of type (2.9). Nevertheless, we will now extend these results to the particular case of reaction waves phenomena. In this context, we are interested in the propagation of steady self-similar waves, i.e. parabolic PDEs of type (1.10) with solution

$$
u(x, t)=u_{0}(x-c t),
$$

where $c$ is the steady speed of the wavefront. 
We have already showed the link between problems (2.9) and (1.10), furthermore a simple dimensionless analysis of traveling waves yield expressions (2.10) and (2.11) with scaling constants $D$ and $k$ (see [33]). Therefore, we can easily show that for an integer $s>0$,

$$
\partial_{x}^{s} V(x)=\partial_{x}^{s} f^{\prime}\left(u_{0}\right)=\partial_{x}^{s-1}\left(f^{\prime \prime}\left(u_{0}\right) \partial_{x} u_{0}\right)
$$

Taking norms and taking into consideration (2.11) under condition $k D=1$, we obtain that

$$
\left\|\partial_{x}^{s} V(x)\right\|_{\infty}=\left\|\partial_{x}^{s} f^{\prime}\left(u_{0}\right)\right\|_{\infty}=\left\|\partial_{x}^{s-1}\left(f^{\prime \prime}\left(u_{0}\right) \partial_{x} u_{0}\right)\right\|_{\infty}=k^{s}\left\|\partial_{x}^{s-1}\left(f^{\prime \prime}\left(u_{0}\right) \partial_{x} \bar{u}_{0}\right)\right\|_{\infty}
$$

where $\bar{u}(x, t)=\bar{u}_{0}(x-c t)$ is a reference solution of (1.10) when $k=1$. Moreover, following (2.10), condition $D k=1$ implies constant speed $c$ for all $k$. Then, we can rewrite Lemma 2.7 as follows.

Lemma 2.9. Consider problem (2.9) with initial conditions $u_{0}$ and $v_{0}$. Furthermore, let us assume that $D k=1$ and that condition (2.33) is satisfied. Denoting by $E_{L}(t)=\mathrm{e}^{t\left(D \partial_{x}^{2}-k f^{\prime}\left(u_{0}\right)\right)}-L(t), E_{S}(t)=\mathrm{e}^{t\left(D \partial_{x}^{2}-k f^{\prime}\left(u_{0}\right)\right)}-S(t)$ and $\tau=k t$, then for a fixed $t>0$ there exist $\kappa_{L}, \kappa_{S}, C_{L}, C_{S} \in \mathbb{R}^{+}$such that we have the following bounds

$$
\left\|E_{L}(t) u_{0}-E_{L}(t) v_{0}\right\|_{\mathrm{L}^{2}} \leq \kappa_{L} C_{L} \tau \sqrt{\tau} \mathrm{e}^{\left\|f^{\prime}\left(u_{0}\right)\right\|_{\infty} \tau}\left\|u_{0}-v_{0}\right\|_{\mathrm{L}^{2}},
$$

and

$$
\left\|E_{S}(t) u_{0}-E_{S}(t) v_{0}\right\|_{L^{2}} \leq \kappa_{S} C_{S} \tau^{2} \mathrm{e}^{\left\|f^{\prime}\left(u_{0}\right)\right\|_{\infty} \tau}\left\|u_{0}-v_{0}\right\|_{\mathrm{L}^{2}} .
$$

Proof. The proof of (2.34) and (2.35) is straightforward to obtain, considering (2.33) and rewriting (2.22) and $(2.23)$ as

$$
\begin{aligned}
\left\|E_{L}(t) u_{0}\right\|_{\mathrm{L}^{2}} & \leq\left(\frac{8}{3} t \sqrt{t}\left(\frac{k\left\|g\left(u_{0}\right)\right\|_{\infty}}{\sqrt{2 e D}}\right)+\frac{t^{2}}{2} k^{2}\left\|\partial_{x} g\left(u_{0}\right)\right\|_{\infty}\right) \mathrm{e}^{k\left\|f^{\prime}\left(u_{0}\right)\right\|_{\infty} t}\left\|u_{0}\right\|_{\mathrm{L}^{2}}, \\
\left\|E_{S}(t) u_{0}\right\|_{\mathrm{L}^{2}} & \leq\left(\frac{t^{2}}{4}\left(\frac{\pi}{e} k^{2}\left\|\partial_{x} g\left(u_{0}\right)\right\|_{\infty}\right)+\frac{t^{3}}{12}\left(k^{3}\left\|g\left(u_{0}\right)\right\|_{\infty}^{2}+D k^{4}\left\|\partial_{x}^{3} g\left(u_{0}\right)\right\|_{\infty}\right)\right) \mathrm{e}^{k\left\|f^{\prime}\left(u_{0}\right)\right\|_{\infty} t\left\|u_{0}\right\|_{\mathrm{L}^{2}}}
\end{aligned}
$$

where $g\left(u_{0}\right)=f^{\prime \prime}\left(u_{0}\right) \partial_{x} \bar{u}_{0}$, then we take $D k=1, \tau=k t$ and redefine $C_{L}$ and $C_{S}$ as

$$
\begin{aligned}
& C_{L}=\max \left(\frac{8\left\|g\left(u_{0}\right)\right\|_{\infty}}{3 \sqrt{2 e}}, \frac{\left\|\partial_{x} g\left(u_{0}\right)\right\|_{\infty}}{2}\right), \\
& C_{S}=\max \left(\frac{\pi\left\|\partial_{x} g\left(u_{0}\right)\right\|_{\infty}}{4 e}, \frac{\left\|g\left(u_{0}\right)\right\|_{\infty}^{2}}{12}, \frac{\left\|\partial_{x}^{3} g\left(u_{0}\right)\right\|_{\infty}}{12}\right) .
\end{aligned}
$$

With this lemma, the following corollary of Theorem 2.8 can be obtained.

Corollary 2.10. Let $\mathcal{F}^{\Delta T}\left(U_{n-1}\right)$ be the exact solution of problem (2.9) on time subdomain $I_{n-1}$. Furthermore, let us assume that $D k=1$ and that condition $(2.33)$ is satisfied. If $\mathcal{G}^{\Delta T}\left(U_{n-1}\right)$ is the Lie approximate solution with local error bounded by (2.34) satisfying (2.24), then at iteration $i$ of the parareal algorithm (1.5), there exist some finite $\kappa_{L}, \kappa_{S}, C_{L}, C_{S}, \Lambda_{T_{n}} \in \mathbb{R}^{+}$such that we have the bound

$$
\left\|u\left(T_{n}\right)-\tilde{U}_{n}^{i}\right\|_{L^{2}} \leq \Lambda_{T_{n}} \frac{\left(\kappa_{L} C_{L} k T_{n}\right)^{i+1}}{(i+1) !}(k \Delta T)^{(i+1) / 2}
$$


with $\Lambda_{T_{n}}=\left\|u_{0}\right\|_{\mathrm{L}^{2}} \mathrm{e}^{\left\|f^{\prime}\left(u_{0}\right)\right\|_{\infty} k T_{n}}$. Otherwise, if $\mathcal{G}^{\Delta T}\left(U_{n-1}\right)$ is the Strang approximate solution with local error bounded by (2.35) satisfying (2.26), then we have the bound

$$
\left\|u\left(T_{n}\right)-\tilde{U}_{n}^{i}\right\|_{L^{2}} \leq \Lambda_{T_{n}} \frac{\left(\kappa_{S} C_{S} k T_{n}\right)^{i+1}}{(i+1) !}(k \Delta T)^{(i+1)}
$$

Proof. The proof comes out directly from Theorem 2.8 and Lemma 2.9, considering $D k=1$ and condition (2.33).

We see that also in this case, all the terms in bounds (2.38) and (2.39) are known or can be calculated for a general problem of type (2.9) satisfying (2.33). Moreover, constants $C_{L}, C_{S}, \kappa_{L}$ and $\kappa_{S}$ have been established in the proof of Lemma 2.9 and this time, $C_{L}$ and $C_{S}$ are completely independent of coefficients $k$ and $D$, so they are valid for any speed/gradient configuration of the wavefront. By the way, from bounds of Lemma 2.9 as well as those of Corollary 2.10, we can consider coefficient $k$ as a time scaling parameter through $\tau=k t$.

From these results we conclude that the convergence rate of the algorithm in the Lie (resp. Strang) case behaves like $k^{1.5}$ (resp. $k^{2}$ ) for a fixed $\Delta T$. And as established by condition (2.11), higher $k$ implies the propagation of wavefronts with higher spatial gradients at the same speed. As a matter of fact, the following corollary establishes more precisely the influence of high spatial gradients onto the performance of the parareal algorithm.

Corollary 2.11. Let us consider Lemma 2.9 and Corollary 2.10. Denoting by $E_{n}^{i}=\left\|u\left(T_{n}\right)-\tilde{U}_{n}^{i}\right\|_{\mathrm{L}^{2}}$ with fixed $k \geq 1$ into (2.9). Then, at iteration $i$ of the parareal algorithm (1.5), there exist some finite $A, B, q \in \mathbb{R}^{+}$such that we have the bound

$$
E_{n}^{i} \leq \mathrm{e}^{A+(i+1) q B} \bar{E}_{n}^{i}
$$

where $\bar{E}_{n}^{i}$ is a fixed reference value of $E_{n}^{i}$ with $k=D=1$ in (2.9).

Proof. From Corollary 2.10, we have

$$
E_{n}^{i} \leq \Lambda_{T_{n}} k^{q(i+1)} \frac{\left(\kappa_{G} C_{G} T_{n}\right)^{i+1}}{(i+1) !} \Delta T^{(q-1)(i+1)},
$$

with $q=3 / 2, C_{G}=C_{L}$ and $\kappa_{G}=\kappa_{L}\left(\right.$ resp. $q=2, C_{G}=C_{S}$ and $\left.\kappa_{G}=\kappa_{S}\right)$ if $\mathcal{G}$ is the Lie (resp. Strang) approximate solution.

After simple computations, we obtain

$$
\ln E_{n}^{i} \leq(k-1)\left\|f^{\prime}\left(u_{0}\right)\right\|_{\infty} T_{n}+q(i+1) \ln k+\ln \bar{E}_{n}^{i}
$$

that yields (2.40) with

$$
A \geq(k-1)\left\|f^{\prime}\left(u_{0}\right)\right\|_{\infty} T_{n} \text { and } B \geq \ln k .
$$

Thus, Corollary 2.11 establishes the bound on the convergence rate of the algorithm for a fixed $k$ into (2.9); as a consequence, the convergence rate is directly related to the value of the highest spatial gradient of the wavefront. In particular, the simulation of sharper wavefronts (higher $k$ ) implies weaker convergence rates since constants $A$ and $B$ into (2.40) increase. This can be easily shown from (2.40) if we consider the line $r_{n}(i)$ :

$$
\ln E_{n}^{i}-\ln \bar{E}_{n}^{i} \leq r_{n}(i)=A+(i+1) q B
$$

where we see that its slope is directly proportional to $\ln k$ through $B \propto \ln k$ for the same approximate solution, i.e. same $q$; and the convergence rate gets lower when sharper spatial gradients are present in the solution.

Now that we have obtained these results that explain the behavior of the parareal algorithm when we consider an operator splitting scheme as the coarse approximation technique, we need to validate them with some numerical examples. This is the goal of the next section. 


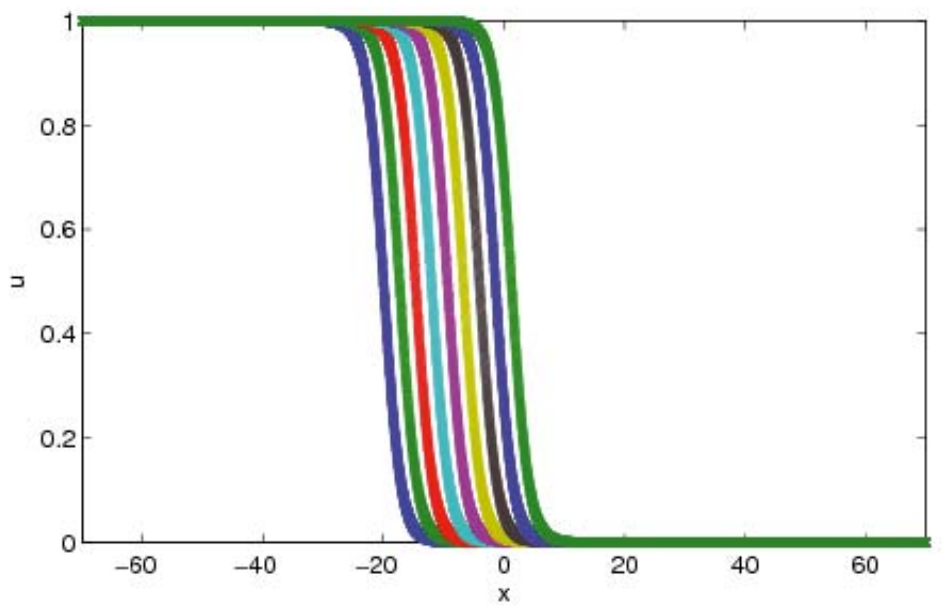

FiguRE 1. Standard KPP traveling wave, discretization with 5001 points on the $[-70,70]$ region. Self-similar solutions for eight time intervals after the initial condition. (Figure in color available online at www.esaim-m2an.org.)

\section{NumericAl SIMUlations}

In this section, we will present some illustrating simulations in order to first, validate the theoretical results presented before, and secondly, to investigate the performance of such algorithms on multi-scale reaction wave phenomena. Both mathematical models considered in the following come from nonlinear chemical dynamics; nevertheless, the conclusions might be extended to similar reaction-diffusion models in other domains. In this way, the 1D KPP model is a clarifying example of the deduced behavior of the parareal splitting technique applied on such type of problems. Then, with a more efficient operator splitting strategy inspired by [21,22], we will consider a much more complex model, the 1D and 2D BZ system, that will also confirm the previous results and will give us some insights into the performance of the algorithm and the alternative strategies for these multi-scale problems.

\subsection{D KPP equation}

Following the theoretical investigations we have presented, we focus in this part on the numerical evidence of the convergence rate reduction associated to splitting solvers in a typical nonlinear framework of stiff traveling waves. Let us first recall the Kolmogorov-Petrovskii-Piskunov model. In their original paper dated in 1937 [41], these authors introduced a model describing the propagation of a virus and the first rigorous analysis of a stable traveling wave solution of a nonlinear reaction-diffusion equation [33,67]. The equation is the following:

$$
\partial_{t} u-D \Delta u=k u^{2}(1-u)
$$

with homogeneous Neumann boundary conditions.

The description of the dimensionless model and the structure of the exact solution can be found in [33]. Thus, the dimensionless analysis shows that in the case of $D=1$ and $k=1$, the velocity of the self-similar traveling wave is $c=1 / \sqrt{2}$ and the maximal gradient value reaches $1 / \sqrt{32}$. The structure of the wave can be observed in Figure 1 with a discretization of 5001 points of the interval $[-70,70]$ and a time varying in $[0,30]$ divided into eight time intervals.

The key point of this illustration is that the velocity of the traveling wave is proportional to $(k D)^{1 / 2}$, whereas the maximal gradient is proportional to $(k / D)^{1 / 2}$. Thus, switching to values $k=10.0$ and $D=0.1$, the velocity 


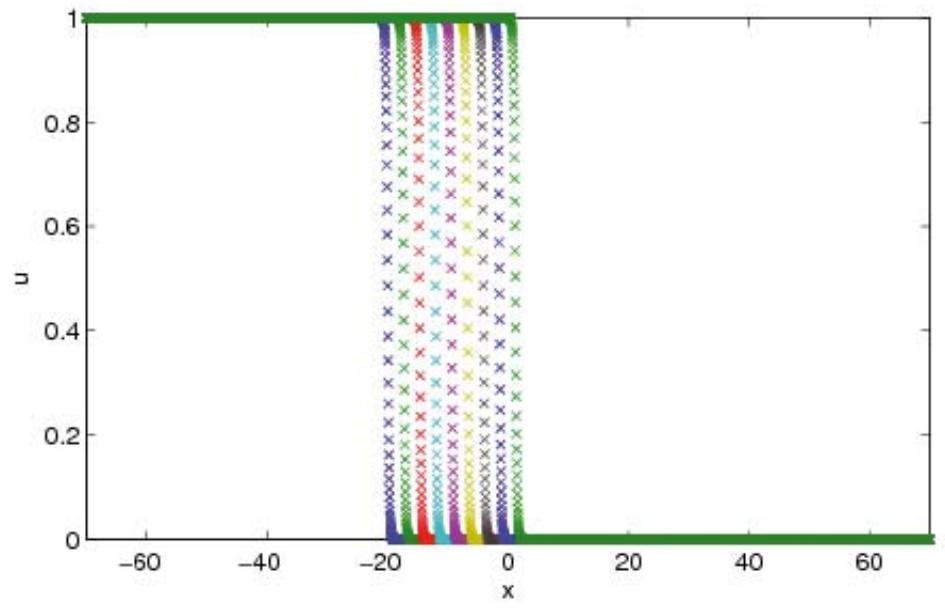

FiguRE 2. "Stiff" KPP traveling wave, discretization with 5001 points on the $[-70,70]$ region. Self-similar solutions for eight time intervals after the initial condition. (Figure in color available online at www.esaim-m2an.org.)

is preserved, but the maximal gradient is multiplied by a factor of 10 and introduces stiffness in the equation, as presented in Figure 2. For the spatial discretizations considered, the wave, however "stiff", is always well solved on the considered grid. This model is then a very suitable example because it coincides perfectly with the general reaction-diffusion structure considered throughout our theoretical study.

Application of the method of lines with a finite difference second order discretization in space implies a discretization of the Laplacian operator in (3.1) and thus, leads to a system of nonlinear ODEs. For the parareal scheme, we then need the fine and coarse temporal integrations of this semi-discretized problem. Based on the previous considerations, we choose a Lie (or Strang) split scheme as the coarse solver and a very accurate Lie (or Strang) split scheme as the fine one, the latter considers smaller splitting time step. In all cases, each of the time integration substeps (reaction and diffusion substeps) is integrated by a unique and very accurate solver in time: LSODE with very fine predefined tolerances. In this way, we decouple the errors originated by the operator splitting itself from the errors coming from the temporal integration of the substeps, in order to remain coherent with the theoretical study conducted in the paper.

We start from an already self-similar solution and evaluate the ability of the parareal solution to reproduce the correct self-similar profile. The splitting time steps for both fine and coarse solvers, are defined such that the wave speed is correctly calculated. Figures 3 and 4 below show the convergence results of the parareal algorithm considering the convergence of the parareal iterative solutions towards the fine solution, computed separately and sequentially by the fine solver. The first iteration corresponds to the initial coarse approximation and the time domain is decomposed into $N=128$ time subdomains.

In Figure 3, on the one hand we observe a clean and fast iterative evolution towards convergence of the Lie splitting technique applied to the standard KPP equation. In fact, only 10 over 128 subdomains have been solved directly by the fine solver to achieve the fine accuracy. On the other hand, a much more lower convergence rate of the algorithm is shown while applying the parareal Lie scheme to the "stiff" KPP equation. In fact, after 15 iterations we are very far from the accuracy obtained in the standard case.

The key point in this paper is the reduction of the convergence rate exhibited by the "stiff" case, where the velocity of the wave is the same, but the maximal gradient is ten times higher. Thus, considering the line defined by (2.41) as a consequence of Corollary 2.11 where $B=1$ since $k=10$ (considering log instead of ln), we have plotted a straight line of slope $q=1.5$ in order to show the very good agreement to the bound predicted by the theory. 


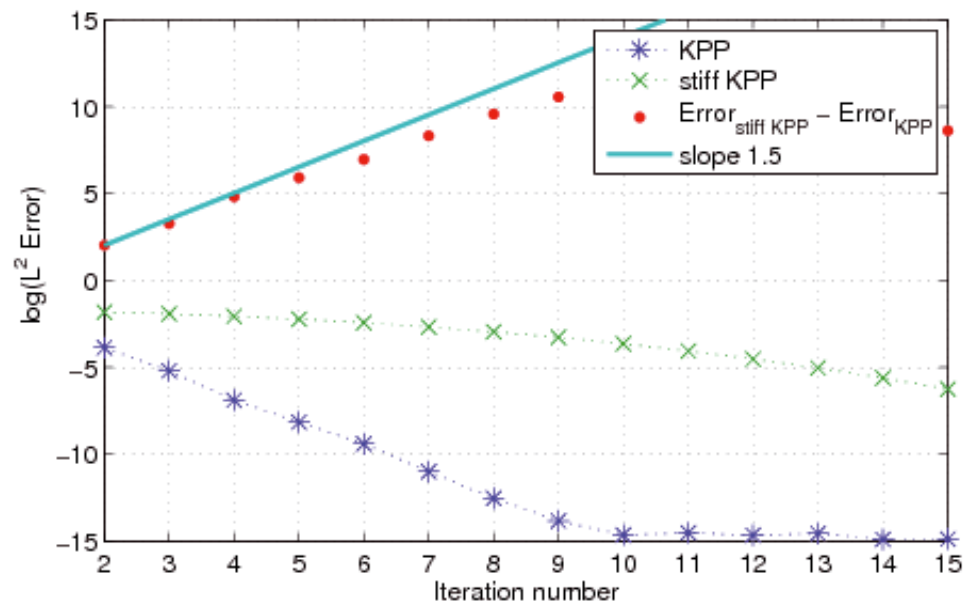

Figure 3. Comparison of convergence rates of the parareal operator splitting at time $t=15$ for 1D KPP equation for the Lie splitting scheme.

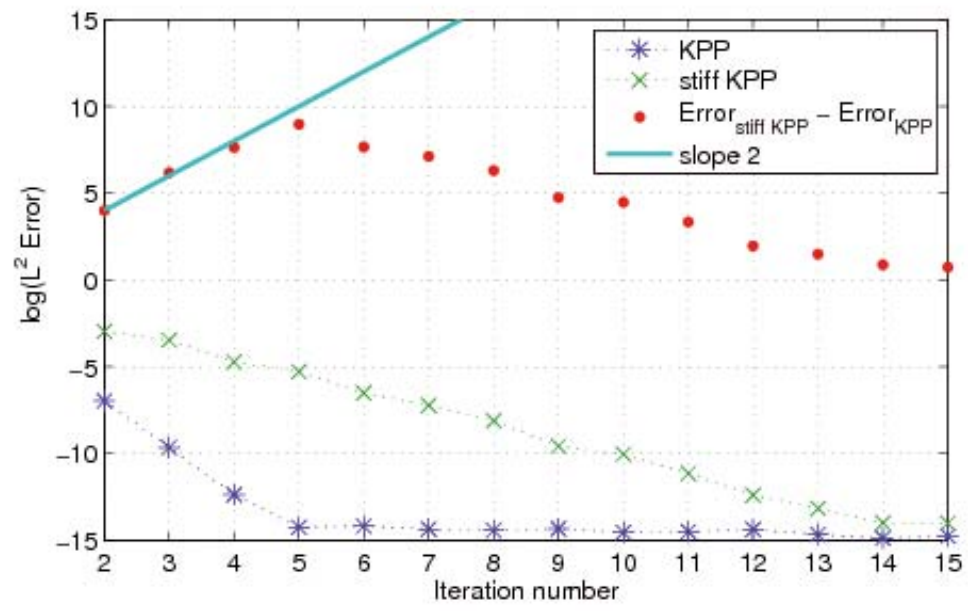

FiguRE 4. Comparison of convergence rates of the parareal operator splitting at time $t=15$ for 1D KPP equation for the Strang splitting scheme.

The same conclusions arise from the Strang case, as it is shown in Figure 4, where this time $q=2$, as established into Corollary 2.11. Notice that the parareal scheme applied to the standard KPP equation converges faster than in the previous Lie case, as a Strang scheme is naturally more accurate that a Lie one. Thus, we obtain less coarse approximations and convergence after five iterations. Notice also that the stiff case practically converges after 15 iterations, which was not the case in the previous Lie application (Fig. 3). Therefore, let us consider a more accurate Lie application where the splitting time steps are smaller so that the stiff case converges completely. Figure 5 describes this situation where once again the theoretical results are validated and for which convergence is achieved after five (resp. 9) iterations for the standard (resp. stiff) case.

Let us finally see what happens with a coarse solver that considers the initial coupled reaction-diffusion system instead of the split one. To limit important computational cost, let us consider an explicit solver such us ROCK4 [1]. This alternative might not be always feasible because of the important stability restrictions 


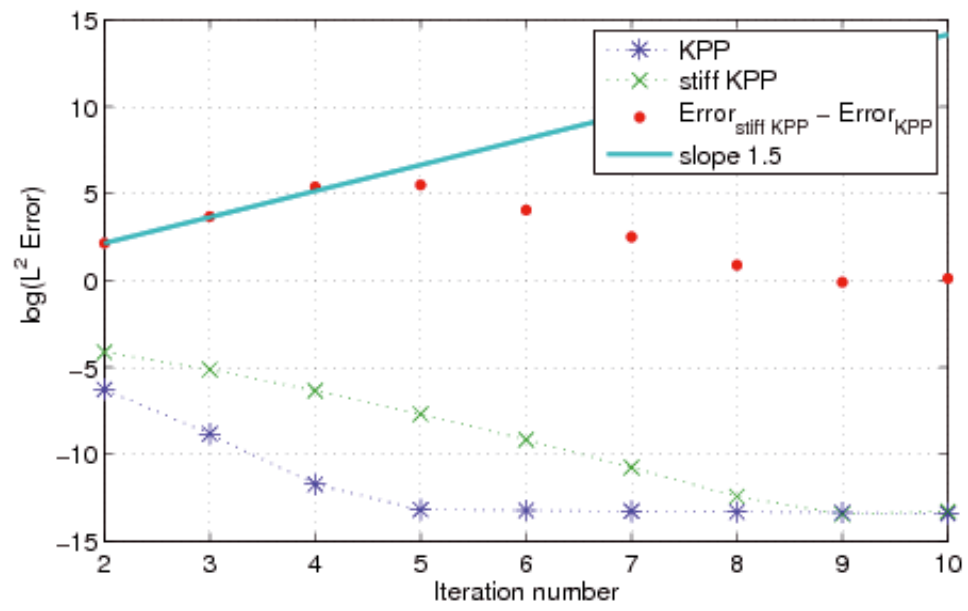

Figure 5. Comparison of convergence rates of the parareal operator splitting at time $t=15$ for 1D KPP equation for a more accurate Lie scheme.

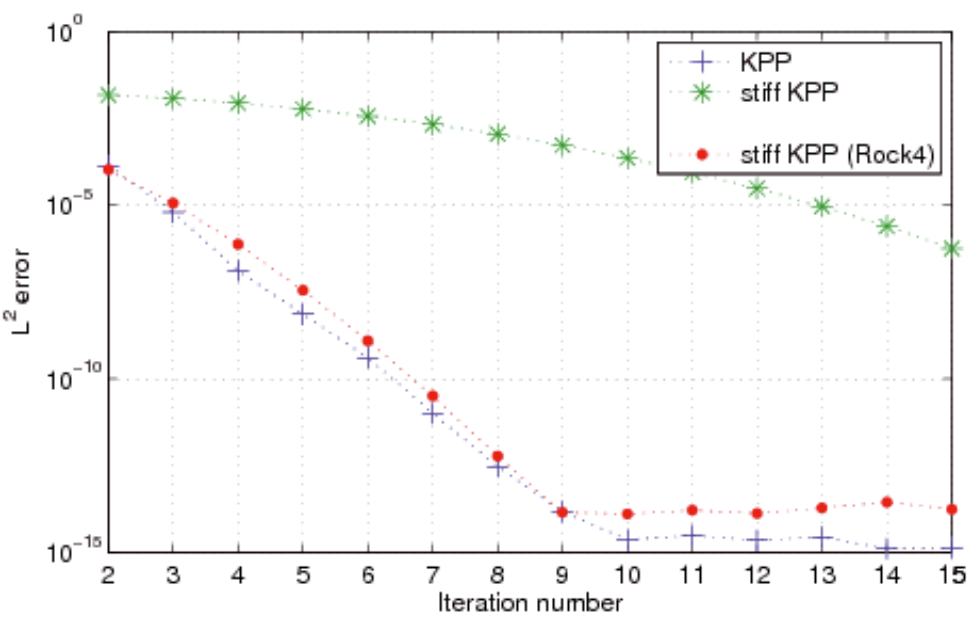

FIGURE 6. Comparison of convergence rates of the parareal algorithm with an operator splitting or ROCK4 as coarse solver, at time $t=15$ for $1 \mathrm{D}$ KPP equation.

of this method when treating problems with important imaginary part eigenvalues (see [1] for more details). We reproduce in Figure 6, the former convergence rates found in the Lie application of Figure 3 for both, the standard and stiff KPP equation, and the new one obtained by the ROCK4-based coarse solver for the stiff case. The latter coupled coarse resolution clearly improves the convergence rate of the parareal scheme for the stiff KPP problem. Hence, we see that an operator splitting approach as coarse approximation clearly implies an important reduction of the convergence rate of the parareal algorithm as it was proven in the previous section.

\subsection{BZ equation}

In this second illustration, we are concerned with the numerical approximation of another model coming from nonlinear chemical dynamics, the Belousov-Zhabotinski reaction, a catalyzed oxidation of an organic species by acid bromated ion (for more details and illustrations, see [24]). We can first consider the two-variable Oregonator 
model, studied in [38]; it has solutions that represent propagation of a steep wavefront by interplay of $\mathrm{HBrO}_{2}$ (hypobromous acid) diffusion with an autocatalytic reaction that quickly generates $\mathrm{HBrO}_{2}$ (using bromide ions $\mathrm{Br}^{-}$as an intermediary species that remains always in equilibrium with local instantaneous $\left.\mathrm{HBrO}_{2}\right)$. Denoting by $b=\left[\mathrm{HBrO}_{2}\right]$ and $c=\left[\mathrm{Br}^{-}\right]$, we consider the following model:

$$
\left.\begin{array}{l}
\frac{\partial b}{\partial \tau}-D_{b} \Delta b=\frac{1}{\varepsilon}\left(b(1-b)+\frac{f(q-b) c}{q+b}\right), \\
\frac{\partial c}{\partial \tau}-D_{c} \Delta c=b-c
\end{array}\right\}
$$

with diffusion coefficients $D_{b}$ and $D_{c}$ and some real positive parameters $f$, small $q$ and small $\varepsilon$.

Nevertheless, a more refined model, introduced in [33] and coming from the classic work of Field, Koros and Noyes (FKN) (1972), takes into account not only the two species $\mathrm{HBrO}_{2}$ and $\mathrm{Br}^{-}$but also the cerium(IV). Denoting by $a=[\mathrm{Ce}(\mathrm{IV})]$, we obtain a very stiff system of three partial differential equations:

$$
\left.\begin{array}{rl}
\frac{\partial a}{\partial \tau}-D_{a} \Delta a & =\frac{1}{\mu}(-q a-a b+f c), \\
\frac{\partial b}{\partial \tau}-D_{b} \Delta b & =\frac{1}{\varepsilon}(q a-a b+b(1-b)), \\
\frac{\partial c}{\partial \tau}-D_{c} \Delta c & =b-c,
\end{array}\right\}
$$

with additional diffusion coefficient $D_{a}$ and real positive parameter $\mu \ll \varepsilon$.

The dynamical systems associated to systems (3.2) and (3.3) model reactive excitable media with a large time scale spectrum (see [33] for more details). Moreover, the spatial configuration with addition of diffusion generates propagating wavefronts with steep spatial gradients. Thus, this model presents all the difficulties associated to a stiff multi-scale configuration. The advantages of applying a splitting strategy to these models have already been studied and presented in [15,21]. In what follows, we will consider the $1 \mathrm{D}$ and $2 \mathrm{D}$ configurations of problem (3.3).

\subsubsection{D BZ equation}

Let us first consider the 1D case of problem (3.3) with homogeneous Neumann boundary conditions and the following parameters (based on [33]): $\varepsilon=10^{-2}, \mu=10^{-5}, f=3$ and $q=2 \times 10^{-4}$, with diffusion coefficients $D_{a}=1, D_{b}=1$ and $D_{c}=0.6$. Following the same partial discretization applied in the previous problem, the structure of the waves can be observed in Figure 7 with a discretization of 4001 points of the interval [0,80] and a time varying in $[0,2]$ divided into eight time intervals.

We then construct an optimal operator splitting configuration already studied and validated [21,22] based on the RDR Strang $S_{2}^{t}$ scheme for which, Radau5 is used for the time integration of the reaction term and ROCK4 for the diffusive part. In this context, Figure 8 shows the iterative evolution and the convergence rate obtained with a RDR Strang operator splitting scheme considered for both fine and coarse solvers, the latter with larger splitting time steps. In the same figure, we see that once again a coupled resolution of the initial reaction-diffusion system (3.3) as coarse solver yields better performances. Also in this illustration, we take ROCK4 as coarse solver of the coupled system.

\subsubsection{D BZ equation}

We now consider the 2D configuration of problem (3.3) with homogeneous Neumann boundary conditions and the following parameters (taken from [15]): $\varepsilon=10^{-2}, \mu=10^{-5}, f=1.6$ and $q=2 \times 10^{-3}$, with diffusion coefficients $D_{a}=2.5 \times 10^{-3}, D_{b}=2.5 \times 10^{-3}$ and $D_{c}=1.5 \times 10^{-3}$. We follow the same partial discretization applied in the previous case and after integration over a time domain of $[0,2]$, we see the developed spiral waves at final time $t=2$ into Figure 9 . 

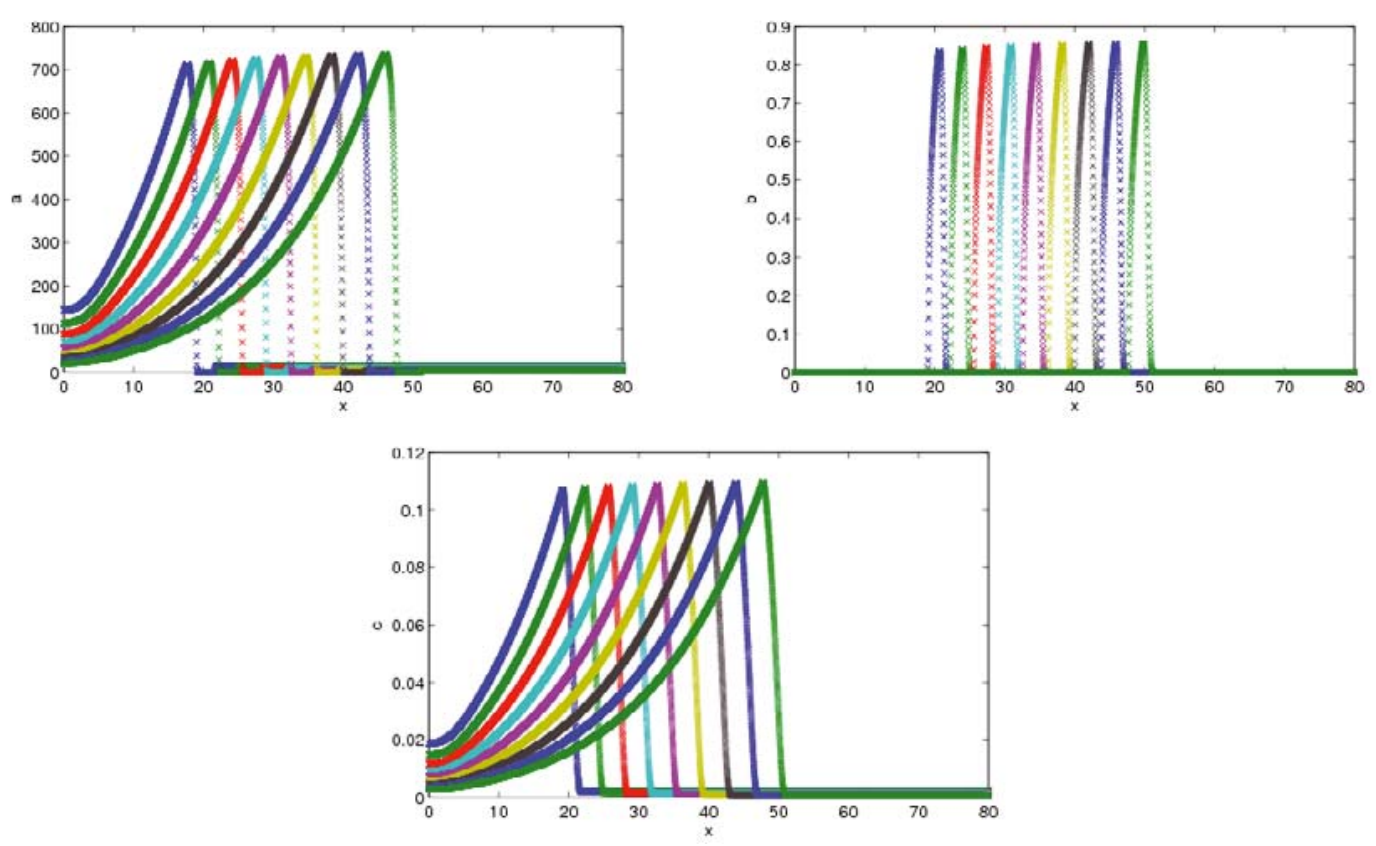

FiguRE 7. 1D BZ traveling waves, discretization with 4001 points on the $[0,80]$ region. Selfsimilar solutions for eight time intervals after the initial condition. (Figure in color available online at www.esaim-m2an.org.)

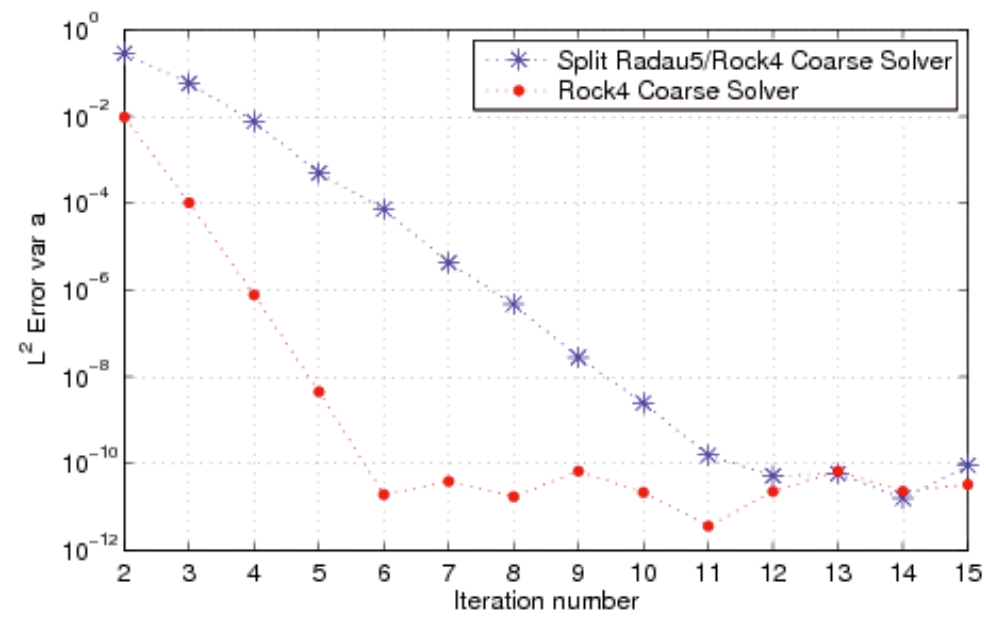

Figure 8. Comparison of convergence rates of the parareal algorithm with an operator splitting or ROCK4 as coarse solver, at time $t=2$ for $1 \mathrm{D}$ BZ equation.

In this illustration, we are concerned with the evaluation of the potential gains obtained in the computational time through a partial parallel computation process. In this way, let us first introduce the following notation:

$T_{\text {fine: }}$ computation time of the fine solver over the whole time domain;

$T_{\text {coarse }}$ : computation time of the coarse solver over the whole time domain; 

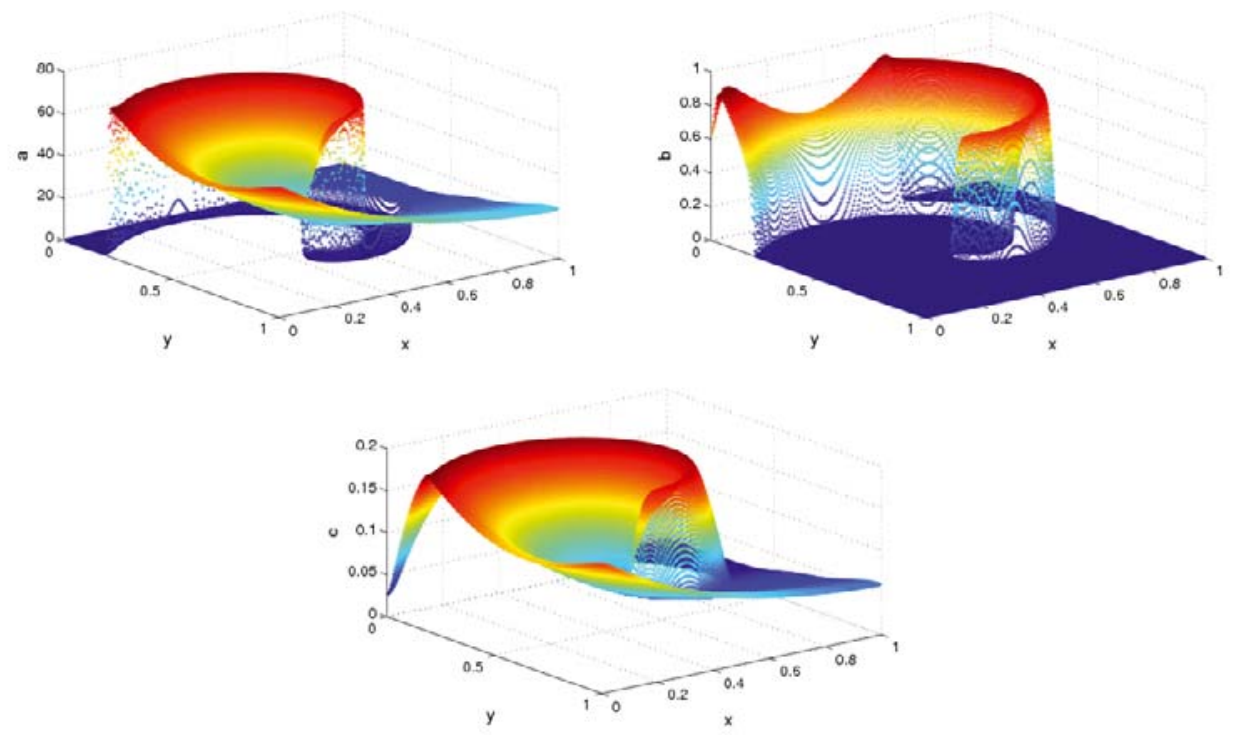

FIGURE 9. 2D BZ spiral waves on a [257 $\times 257]$ grid at $t=2$. (Figure in color available online at www.esaim-m2an.org.)

$N_{\text {proc: }}$ number of processors, i.e. number of time subdomains considered; and

$N_{\text {ite }}$ : number of iterations.

In a parallel environment, the computation time utilized by the parareal algorithm, $T_{\text {para }}$, should be equivalent to the time needed by the fine resolution divided by the number of processors available, that is

$$
T_{\text {para }} \approx N_{\text {ite }} \times \frac{T_{\text {fine }}}{N_{\text {proc }}},
$$

taking into account the iterative procedure. We set this estimate as the optimal ratio, when coarse approximations are very fast. Nevertheless, a more realistic estimate should take into account all coarse approximations computed sequentially in order to initialize each time subdomain. Then, we have

$$
T_{\text {para }} \approx\left(N_{\text {ite }}+1\right) \times T_{\text {coarse }}+N_{\text {ite }} \times \frac{T_{\text {fine }}}{N_{\text {proc }}},
$$

with the initial coarse approximation needed to start the algorithm. Thus, the choice of the coarse solver is crucial and represents the major constraint on the success of the applications.

In this context, let us make some computations using the RDR Strang $S_{2}^{t}$ operator splitting established in the previous $1 \mathrm{D}$ case and ROCK4, as coarse solvers for two different grids, [129 × 129] and [257 × 257], where the time domain has been decomposed into $N=N_{\text {proc }}=64$ time subdomains. Naturally, the fine solver is an accurate RDR Strang operator splitting. According to the parareal scheme, this fine resolution is performed on a cluster made of 32 nodes with 2 processors AMD Opteron 64 bits dual core with speed 2.4 GHz; the numerical code is written in Fortran 95 and uses the MPI library for the parallel features. The results related to each configuration are summarized in Table 1, where the stopping criterion is based on the same order of difference between successive parareal solutions for both configurations. Notice that the splitting scheme is faster than a coupled resolution even if an explicit method such as ROCK4 has been utilized. Nevertheless, the coupled computation as coarse approximation allows faster convergence and thus, a better performance of the algorithm.

This is also reflected in Table 2 where the computation time ratios have been estimated. $N_{\text {proc }} / N_{\text {ite }}$ then represents the optimal ratio described into expression (3.4): 16 for the split coarse solver and 32 for the coupled 
TABLE 1. Computation time in seconds, 2D BZ.

\begin{tabular}{|c|c|c|c|c|}
\hline Grid & \multicolumn{2}{|c|}{$129 \times 129$} & \multicolumn{2}{c|}{$257 \times 257$} \\
\hline Coarse solver & RDR Strang & ROCK4 & RDR Strang & ROCK4 \\
\hline$T_{\text {fine }}$ & 2769.94 & 2757.52 & 11291.36 & 11149.42 \\
\hline$T_{\text {coarse }}$ & 228.53 & 256.07 & 1006.62 & 1177.89 \\
\hline$T_{\text {fine }} / T_{\text {coarse }}$ & 12.12 & 10.77 & 11.22 & 9.47 \\
\hline$N_{\text {ite }}$ & 4 & 2 & 4 & 2 \\
\hline$T_{\text {para }}$ & 1279.81 & 860.30 & 5581.32 & 3869.51 \\
\hline
\end{tabular}

TABle 2. Computation time ratios, 2D BZ.

\begin{tabular}{|c|c|c|c|c|}
\hline Grid & \multicolumn{2}{|c|}{$129 \times 129$} & \multicolumn{2}{c|}{$257 \times 257$} \\
\hline Coarse solver & RDR Strang & ROCK4 & RDR Strang & ROCK4 \\
\hline$N_{\text {proc }}$ & \multicolumn{3}{|c|}{64} \\
\hline$N_{\text {proc }} / N_{\text {ite }}$ & 16 & 32 & 16 & 32 \\
\hline$T_{\text {fine }} / T_{\text {para }}$ & 2.16 & 3.21 & 2.02 & 2.88 \\
\hline
\end{tabular}

one. Hence, the final ratios obtained $(\sim 2-3)$ reflect on the one hand, the important cost of the coarse solvers for stiff problems, and on the other hand, the reduction of convergence rate for an operator splitting scheme as coarse propagator, previously deduced.

\section{Conclusions}

In this paper, we have presented a new numerical strategy that couples an optimal operator splitting technique for multi-scale problems and the parareal algorithm, a promising time parallelization scheme. Considering the state of the art of the literature, we have then conducted a detailed numerical analysis of such techniques in order to introduce complementary and necessary characterization of the numerical behavior of operator splitting and parareal schemes in the context of multi-scale reaction waves. In particular, a precise representation of the impact of stiff configurations on the performance of the algorithm has been mathematically proven for reaction-diffusion models with large spatial gradients.

Then, a set of numerical illustrations have on the one hand, validated the previous theoretical results and explicitly showed the reduction of the convergence rate of the parareal algorithm when an operator splitting is performed for the coarse approximations of the method. And on the other hand, they have highlighted the need of efficient coarse solvers for stiff problems in order to construct more competitive methods.

Finally, it was also numerically demonstrated that a more suitable strategy considers a coupled resolution of the initial reaction-diffusion system as coarse solver. However, this alternative may not always be feasible and other approaches should be evaluated. For instance, the resolution of the reduced model of the BZ system or the set of slow variables for a general multi-scale problem could be considered as a coarse approximation of the complete fine model. Nevertheless, further studies that go out of the scope of this work are necessary in order to test such alternatives; this is the topic of our current research.

Acknowledgements. This research was supported by a fundamental project grant from ANR (French National Research Agency - ANR CIS) PITAC (Project leader Y. Maday), and by a CNRS Ph.D. grant for M. Duarte from the Mathematics (INSMI) and Engineering (INSIS) Institutes of CNRS. This work was granted access to the HPC resources of IDRIS (CNRS Institute of Scientific Computing) under the allocation 2009-i2009066173 made by GENCI (Grand Equipement National de Calcul Intensif) where some of the simulations were performed. The authors are grateful to Stéphane de Chaisemartin, Thierry Dumont, Violaine Louvet and Frédérique Laurent for valuable discussions and important assistance throughout this project. 


\section{REFERENCES}

[1] A. Abdulle, Fourth order Chebyshev methods with recurrence relation. J. Sci. Comput. 23 (2002) 2041-2054.

[2] G. Akrivis, M. Crouzeix and C. Makridakis, Implicit-explicit multistep finite element methods for nonlinear parabolic problems. Math. Comp. 67 (1998) 457-477.

[3] L. Baffico, S. Bernard, Y. Maday, G. Turinici and G. Zérah, Parallel-in-time molecular-dynamics simulations. Phys. Rev. E 66 (2002) 1-4.

[4] G. Bal, On the convergence and the stability of the parareal algorithm to solve partial differential equations, in Proceedings of the 15th International Domain Decomposition Conference, Lect. Notes Comput. Sci. Eng. 40, Springer, Berlin (2003) $426-432$.

[5] G. Bal and Y. Maday, A "parareal" time discretization for non-linear PDE's with application to the pricing of an American put, in Recent Developments in Domain Decomposition Methods, Lect. Notes Comput. Sci. Eng. 23, Springer, Berlin (2003) 189-202.

[6] D. Barkley, A model for fast computer simulation of waves in excitable media. Physica D 49 (1991) 61-70.

[7] P. Chartier and B. Philippe, A parallel shooting technique for solving dissipative ODEs. Computing 51 (1993) $209-236$.

[8] Y. D'Angelo, Analyse et Simulation Numérique de Phénomènes liés à la Combustion Supersonique. Ph.D. thesis, École Nationale des Ponts et Chaussées, France (1994).

[9] Y. D'Angelo and B. Larrouturou, Comparison and analysis of some numerical schemes for stiff complex chemistry problems. RAIRO Modél. Math. Anal. Numér. 29 (1995) 259-301.

[10] M.S. Day and J.B. Bell, Numerical simulation of laminar reacting flows with complex chemistry. Combust. Theory Modelling 4 (2000) 535-556.

[11] S. Descombes, Convergence of a splitting method of high order for reaction-diffusion systems. Math. Comp. 70 (2001) 14811501.

[12] S. Descombes and T. Dumont, Numerical simulation of a stroke: Computational problems and methodology. Prog. Biophys. Mol. Biol. 97 (2008) 40-53.

[13] S. Descombes and M. Massot, Operator splitting for nonlinear reaction-diffusion systems with an entropic structure: Singular perturbation and order reduction. Numer. Math. 97 (2004) 667-698.

[14] S. Descombes and M. Schatzman, Strang's formula for holomorphic semi-groups. J. Math. Pures Appl. 81 (2002) 93-114.

[15] S. Descombes, T. Dumont and M. Massot, Operator splitting for stiff nonlinear reaction-diffusion systems: Order reduction and application to spiral waves, in Patterns and waves (Saint Petersburg, 2002), AkademPrint, St. Petersburg (2003) 386-482.

[16] S. Descombes, T. Dumont, V. Louvet and M. Massot, On the local and global errors of splitting approximations of reactiondiffusion equations with high spatial gradients. Int. J. Computer Mathematics 84 (2007) 749-765.

[17] S. Descombes, T. Dumont, V. Louvet, M. Massot, F. Laurent and J. Beaulaurier, Operator splitting techniques for multi-scale reacting waves and application to low mach number flames with complex chemistry: Theoretical and numerical aspects. In preparation (2011).

[18] P. Deuflhard, A modified Newton method for the solution of ill-conditioned systems of nonlinear equations with application to multiple shooting. Numer. Math. 22 (1974) 289-315.

[19] P. Deufhard, Newton Methods for Nonlinear Problems - Affine invariance and adaptive algorithms. Springer-Verlag (2004).

[20] M. Dowle, R.M. Mantel and D. Barkley, Fast simulations of waves in three-dimensional excitable media. Int. J. Bif. Chaos 7 (1997) 2529-2545.

[21] M. Duarte, M. Massot, S. Descombes, C. Tenaud, T. Dumont, V. Louvet and F. Laurent, New resolution strategy for multiscale reaction waves using time operator splitting, space adaptive multiresolution and dedicated high order implicit/explicit time integrators. J. Sci. Comput. (to appear) available on HAL (http://hal.archives-ouvertes.fr/hal-00457731).

[22] T. Dumont, M. Duarte, S. Descombes, M.A. Dronne, M. Massot and V. Louvet, Simulation of human ischemic stroke in realistic 3D geometry: A numerical strategy. Bull. Math. Biol. (to appear) available on HAL (http://hal. archives-ouvertes. fr/hal-00546223).

[23] T. Echekki, Multiscale methods in turbulent combustion: Strategies and computational challenges. Computational Science $\mathcal{E}$ Discovery 2 (2009) 013001.

[24] I.R. Epstein and J.A. Pojman, An Introduction to Nonlinear Chemical Dynamics - Oscillations, Waves, Patterns and Chaos. Oxford University Press (1998).

[25] C. Farhat and M. Chandesris, Time-decomposed parallel time-integrators: Theory and feasibility studies for fluid, structure, and fluid-structure applications. Int. J. Numer. Methods Eng. 58 (2003) 1397-1434.

[26] F. Fischer, F. Hecht and Y. Maday, A parareal in time semi-implicit approximation of the Navier-Stokes equations, in Proceedings of the 15th International Domain Decomposition Conference, Lect. Notes Comput. Sci. Eng. 40, Springer, Berlin (2003) 433-440.

[27] M. Gander and E. Hairer, Nonlinear convergence analysis for the parareal algorithm, in Domain Decomposition Methods in Science and Engineering XVII, Springer, Berlin (2008) 45-56.

[28] M. Gander and S. Vandewalle, Analysis of the parareal time-parallel time-integration method. J. Sci. Comput. 29 (2007) $556-578$. 
[29] I. Garrido, M.S. Espedal and G.E. Fladmark, A convergence algorithm for time parallelization applied to reservoir simulation, in Proceedings of the 15th International Domain Decomposition Conference, Lect. Notes Comput. Sci. Eng. 40, Springer, Berlin (2003) 469-476.

[30] I. Garrido, B. Lee, G.E. Fladmark and M.S. Espedal, Convergent iterative schemes for time parallelization. Math. Comput. 75 (2006) 1403-1428.

[31] V. Giovangigli, Multicomponent flow modeling. Birkhäuser Boston Inc., Boston, MA (1999).

[32] S.A. Gokoglu, Significance of vapor phase chemical reactions on cvd rates predicted by chemically frozen and local thermochemical equilibrium boundary layer theories. J. Electrochem. Soc. 135 (1988) 1562-1570.

[33] P. Gray and S.K. Scott, Chemical oscillations and instabilites. Oxford University Press (1994).

[34] E. Grenier, M.A. Dronne, S. Descombes, H. Gilquin, A. Jaillard, M. Hommel and J.P. Boissel, A numerical study of the blocking of migraine by Rolando sulcus. Prog. Biophys. Mol. Biol. 97 (2008) 54-59.

[35] E. Hairer and G. Wanner, Solving ordinary differential equations II - Stiff and differential-algebraic problems. Second edition, Springer-Verlag, Berlin (1996).

[36] E. Hairer, C. Lubich and G. Wanner, Geometric Numerical Integration - Structure-Preserving Algorithms for Odinary Differential Equations. Second edition, Springer-Verlag, Berlin (2006).

[37] W. Hundsdorfer and J.G. Verwer, Numerical Solution of Time-Dependent Advection-Diffusion-Reaction Equations. SpringerVerlag, Berlin (2003).

[38] W. Jahnke, W.E. Skaggs and A.T. Winfree, Chemical vortex dynamics in the Belousov-Zhabotinsky reaction and in the two-variable Oregonator model. J. Phys. Chem. 93 (1989) 740-749.

[39] J. Kim and S.Y. Cho, Computation accuracy and efficiency of the time-splitting method in solving atmosperic transportchemistry equations. Atmos. Environ. 31 (1997) 2215-2224.

[40] O.M. Knio, H.N. Najm and P.S. Wyckoff, A semi-implicit numerical scheme for reacting flow. II. Stiff, operator-split formulation. J. Comput. Phys. 154 (1999) 467-482.

[41] A.N. Kolmogoroff, I.G. Petrovsky and N.S. Piscounoff, Étude de l'équation de la diffusion avec croissance de la quantité de matière et son application a un problème biologique. Bulletin de l'Université d'état Moscou, Série Internationale Section A Mathématiques et Mécanique 1 (1937) 1-25.

[42] J.L. Lions, Y. Maday and G. Turinici, Résolution d'EDP par un schéma en temps "pararéel". C. R. Acad. Sci. Paris Sér. I Math. 332 (2001) 661-668.

[43] C. Lubich, On splitting methods for Schrödinger-Poisson and cubic nonlinear Schrödinger equations. Math. Comp. 77 (2008) $2141-2153$.

[44] Y. Maday and G. Turinici, A parareal in time procedure for the control of partial differential equations. C. R., Math. 335 (2002) 387-391.

[45] Y. Maday and G. Turinici, The parareal in time iterative solver: A further direction to parallel implementation, in Proceedings of the 15th International Domain Decomposition Conference, Lect. Notes Comput. Sci. Eng. 40, Springer, Berlin (2003) 441-448.

[46] G.I. Marchuk, Splitting and alternating direction methods, in Handbook of numerical analysis I, North-Holland, Amsterdam (1990) 197-462.

[47] M. Massot, Singular perturbation analysis for the reduction of complex chemistry in gaseous mixtures using the entropic structure. Discrete Contin. Dyn. Syst. Ser. B 2 (2002) 433-456.

[48] G.J. McRae, W.R. Goodin and J.H. Seinfeld, Numerical solution of the atmospheric diffusion equation for chemically reacting flows. J. Comput. Phys. 45 (1982) 1-42.

[49] H.N. Najm and O.M. Knio, Modeling Low Mach number reacting flow with detailed chemistry and transport. J. Sci. Comput. 25 (2005) 263-287.

[50] H.N. Najm, P.S. Wyckoff and O.M. Knio, A semi-implicit numerical scheme for reacting flow. I. Stiff chemistry. J. Comput. Phys. 143 (1998) 381-402.

[51] M. Schatzman, Toward non commutative numerical analysis: High order integration in time. J. Sci. Comput. 17 (2002) $107-125$.

[52] L.F. Shampine, B.P. Sommeijer and J.G. Verwer, IRKC: An IMEX solver for stiff diffusion-reaction PDEs. J. Comput. Appl. Math. 196 (2006) 485-497.

[53] M.D. Smooke, Error estimate for the modified Newton method with applications to the solution of nonlinear, two-point boundary value problems. J. Optim. Theory Appl. 39 (1983) 489-511.

[54] B.P. Sommeijer, L.F. Shampine and J.G. Verwer, RKC: An explicit solver for parabolic PDEs. J. Comput. Appl. Math. 88 (1998) 315-326.

[55] B. Sportisse, Contribution à la modélisation des écoulements réactifs : Réduction des modèles de cinétique chimique et simulation de la pollution atmosphérique. Ph.D. thesis, École Polytechnique, France (1999).

[56] B. Sportisse, An analysis of operator splitting techniques in the stiff case. J. Comput. Phys. 161 (2000) 140-168.

[57] B. Sportisse and R. Djouad, Reduction of chemical kinetics in air pollution modeling. J. Comput. Phys. 164 (2000) 354-376.

[58] G.A. Staff and E.M. Rønquist, Stability of the parareal algorithm, in Proceedings of the 15th International Domain Decomposition Conference, Lect. Notes Comput. Sci. Eng. 40, Springer, Berlin (2003) 449-456. 
[59] G. Strang, Accurate partial difference methods. I. Linear Cauchy problems. Arch. Ration. Mech. Anal. 12 (1963) $392-402$.

[60] G. Strang, On the construction and comparison of difference schemes. SIAM J. Numer. Anal. 5 (1968) 506-517.

[61] P. Sun, A pseudo non-time splitting method in air quality modeling. J. Comp. Phys. 127 (1996) 152-157.

[62] R. Témam, Sur l'approximation de la solution des équations de Navier-Stokes par la méthode des pas fractionnaires. I. Arch. Rational Mech. Anal. 32 (1969) 135-153.

[63] R. Témam, Sur l'approximation de la solution des équations de Navier-Stokes par la méthode des pas fractionnaires. II. Arch. Rational Mech. Anal. 33 (1969) 377-385.

[64] J.G. Verwer and B.P. Sommeijer, An implicit-explicit Runge-Kutta-Chebyshev scheme for diffusion-reaction equations. SIAM J. Sci. Comput. 25 (2004) 1824-1835.

[65] J.G. Verwer and B. Sportisse, Note on operator splitting in a stiff linear case. Rep. MAS-R9830 (1998).

[66] J.G. Verwer, B.P. Sommeijer and W. Hundsdorfer, RKC time-stepping for advection-diffusion-reaction problems. J. Comput. Phys. 201 (2004) 61-79.

[67] A.I. Volpert, V.A. Volpert and V.A. Volpert, Traveling wave solutions of parabolic systems. American Mathematical Society, Providence, RI (1994).

[68] N.N. Yanenko, The method of fractional steps. The solution of problems of mathematical physics in several variables. SpringerVerlag, New York (1971). 